\title{
Confluence operators and their relationships with revision, update and merging
}

\author{
Sébastien Konieczny • Ramon Pino Pérez
}

(C) Springer Science+Business Media Dordrecht 2013

\begin{abstract}
In this paper we introduce confluence operators, that are inspired by the existing links between belief revision, update and merging operators. Roughly, update operators can be considered as pointwise revision, whereas revision operators can be considered as special case of merging operators. Confluence operators are to merging operators what update operators are to revision operators. Similarly, update operators can be considered as special case of confluence operators just as revision can be considered as special case of merging operators. Confluence operators gives all possible agreement situations from a set of belief bases.
\end{abstract}

Keywords Belief dynamics $\cdot$ Belief revision - Update $\cdot$ Merging $\cdot$ Confluence

Mathematics Subject Classifications (2010) $\quad$ 03B 42 • 68T27 • 68T30

\section{Introduction}

Representing and modeling belief dynamics is a very important issue in Artificial Intelligence, in particular modeling the behaviour of an intelligent autonomous agent. Actually, every autonomous agent has to use a belief base to model a view of the world. The information an agent possesses is precious since it can be costly to

A preliminary version of this work was published in [22]. This paper is an extended and revised version of this work.

S. Konieczny $(\bowtie)$

Centre de Recherche en Informatique de Lens (CRIL), CNRS UMR 8188, Université d'Artois, Lens, France

e-mail: konieczny@cril.fr

R. Pino Pérez

Centro Interdisciplinario de Lógica y Álgebra (CILA), Departamento de Matemáticas,

Facultad de Ciencias, Universidad de Los Andes, Mérida, Venezuela

e-mail: pino@ula.ve 
obtain and since it is necessary to carry on reasoning tasks or to take the appropriate decisions. So, a first class requirement in order to design intelligent autonomous agents is to try to provide her the means to obtain, and to maintain the most faithful belief base. In particular an agent has to be able to incorporate new pieces of information, and to correct the incorrect beliefs when she detect them. Therefore the information dynamics is one of the major components of any autonomous agent.

Common belief change operators are revision operators $[2,16]$, update operators [17] and merging operators [20]. In order to give a quick overview of these operators, let us briefly describe these operators informally, and let us illustrate each operator with an example of the kind of situation that this operator is supposed to model:

- Revision operators aim to model the process of accommodating a new piece of evidence that is more reliable than the current beliefs of the agent. In belief revision the world is static, it is the beliefs of the agents that evolve.

Example 1 (Revision) John knew Linda ${ }^{1}$ with both were PhD students in Philosophy in a very prestigious university. He remembers Linda's activism in feminism and her brilliant record. Both obtain their $\mathrm{PhD}$ degree at the same time. Since then, five years after, John has no news from Linda. However, he thinks that Linda is for sure an activist in feminism and that she occupies an excellent position in a Philosophy Department of one prestigious university. John met Peter, a common classmate, who says him that, surprisingly, Linda is now a bank teller. With this new piece of information John revise his beliefs and he thinks now that Linda is a bank teller who keeps her feminist activism.

- Update operators aim to model the process of the information evolution in a changing world. In belief update the new piece of evidence denotes a change in the world. The world is dynamic, and those (observed) changes modify the beliefs of the agent.

Example 2 (Update) Suppose we have five books, $B_{1}, B_{2}, \ldots, B_{5}$ in a room. ${ }^{2}$ There is also a table in the room. The books may be on or of the table. We know that either $B_{1}$ is the only book on the table or only $B_{3}, B_{4}$ and $B_{5}$ are on the table. A robot comes to the room with the instruction to put all the books on the table or remove all the books from the table. In such a case using update leads to the following belief base: all the books are on the table or there are no books on the table. But if one uses a (common) revision operator ${ }^{3}$ on this example, the result will be that there are no books on the table.

- Merging operators aim to model the process of finding the beliefs which can be associated to a group of agents, from the beliefs of each agent of this group. As for belief revision, the world is considered static, the problem is the combination of different pieces of information about this (static) world.

\footnotetext{
${ }^{1}$ This story is inspired in a famous example in Cognitive Psychology of an experiment by Tversky and Kahneman [31].

${ }^{2}$ This example is taken of [17]

${ }^{3}$ Dalal revision operator [7].
} 
Fig. 1

Revision—update—merging

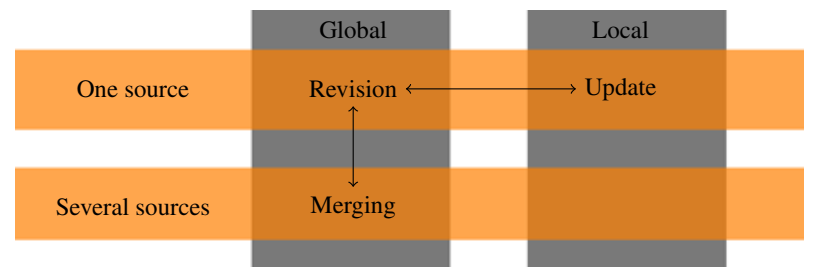

Example 3 (Merging) Margot believes that dolphins are mammals. Sofia believes that dolphins are fishes and that someone that has a sonar system can move without problems in the dark. Agathe believes that dolphins are mammals and that they have a sonar system. By merging these beliefs, it seems sensible for the group to accept as beliefs that dolphins have a sonar system, and can move in the dark. There is some conflict about the fact that dolphins are mammals or fishes. But if one uses majority to solve this conflict, the group then accepts that dolphins are mammals.

Of course, they are various representation languages one can use in order to represent the beliefs, goals, desires, etc. of an agent. One way that has proved very useful is propositional logic. We focus on this case in this work and we present the now classical belief revision operators of the Alchourrón, Gärdenfors amd Makinson (AGM) framework [2] in finite propositional logic in the style of Katsuno and Mendelzon [16]. This approach has the advantage of allowing a clear comparison between different kinds of operators defined in the area of belief dynamics. Another important advantage of this approach is to yield semantical representation theorems which, from one hand, gives a deep understanding of operators and, from the other hand, gives constructive methods for building operators.

Until now, one may have noticed some differences and similarities between these operators. One of the main goals of this work is to establish in a clear and distinct manner the relationships between them. Actually, this will be particularly clear when looking at the technical definitions and representations theorems. There are close relationship between revision [2, 10, 16] and KM update operators [17]. The first ones looking at the beliefs of the agents globally, the second ones looking at them locally $^{4}$ (this sentence will be made formally clear in the paper).

There is also a close connection between revision and merging operators. In fact revision operators can be seen as particular cases of merging operators [20]. These links are represented in Fig. 1.

Looking at Fig. 1 one natural question that naturally comes to mind is to wonder if there is some operation that can fill the empty square (Several sources $\times$ Local). So basically we are looking at an operator that is to merging what update is to revision. Or, the other way round, for an operator that is to update what merging is to revision. We call confluence such operators. Their precise definition, the study of their properties and interrelations with other operators will be the goals of this work.

Let us illustrate the need of such confluence operators on an example.

${ }^{4}$ See $[9,14,23]$ for more discussions on update and its links with revision. 
Example 4 (Confluence) Mary and Peter are planning to buy a car. Mary does not want a German car nor an expensive car. She likes small cars. Peter hesitates between a German, expensive but small car or a car which is not German, nor expensive and is a big car. Taking three propositional variables German_car, Expensive_car and Small_car in this order, Mary's desires are represented by $\bmod (A)=\{001\}$ and Peter's desires by $\bmod (B)=\{111,000\}$. Unlike the fusion operators, the confluence operators will take into account the disjunctive nature of Peter's desires in a fine way. The answer will be $\{001,000,011,101\}$, i.e., the disjunction of a small car not German and not expensive, a big car not German and not expensive, an expensive small car not German and finally a German and small car not expensive. Actually most fusion operators give as result in this case $\{001,000\}$ i.e., a cheap small car which is not German or a cheap big car which is not German. That is exactly the same answer as if Peter's desire about the car is only not German, not expensive and a big car (\{000\}). This is ok if Peter later on solves his hesitation and decides on $\{000\}$. But what happens if Peter finally solves his hesitation by deciding $\{001\}$ ? Merging does not allow to consider this possibility. But confluence operators take into account that Peter's desire about the car might also be/become an expensive and small German car ( $\{111\})$. In this case it would be more appropriate to give also as result a small car that is cheap or not German but not the two at the same time (in symbols $\{011,101\}$ ). Thus, what confluence do is to put on the table all the possibilities when taking into account that Peter's desire can become more precise. In this context, the local aspect have to be thought in the following way: if one agent makes more precise its desires/goals/beliefs this will be the case of the confluence.

We therefore propose in this paper the definition of confluence operators. We propose a set of postulates for confluence operators, and a representation theorem in terms of pre-orders on interpretations. We study the (expected) links between confluence, merging, and update operators. We provide a constructive way to define confluence operators, illustrate their behaviour on an example, and finally we discuss some perspectives of future work on the use of these operators in conciliation processes.

This example shows the typical use of confluence operators: such operators have to be used when we want to combine information (beliefs/goals) coming from several sources, when this information can still evolve in the future, either because the different agent can still change their mind (by refining their base), or because we only have at the moment of the combination an approximation (i.e., a logically weaker view) of their base. Confluence operators allow to not discard any potential combination result.

Update operators can be considered as a local version of revision operators. Revision operators act globally on the base, whereas update operators act locally, model by model. One consequence of this is that if $K_{1}$ implies $K_{2}$, then the result of the update of the $K_{1}$ by a new piece of information $\mu$ will imply the result of the update of the $K_{2}$ by $\mu$. Whereas this property does not hold for revision. Similarly Confluence operators are a local version of Merging operators. We will see in Section 3.1 (Corollary 1) that there is the same kind of distinction for confluence with respect to merging: If a profile $E_{1}$ implies $E_{2}$, then the confluence of $E_{1}$ will implies the confluence of $E_{2}$, where there is no such property with merging. 
The structure of this paper is as follows. Section 2 introduces the needed notations and the definitions of revision, update and merging operators. We recall there briefly the respective representation theorems in terms of pre-orders on interpretations. We give also in this section some relationships between revision, update and merging operators. Section 3 introduces formally the confluence operators. We establish a representation theorem for these operators, and we show some links between confluence and update and merging operators. Section 4 introduces a family of confluence operators based on distances, and illustrate their behaviour on a detailed example. In Section 5 we end with some concluding remarks and some perspectives of future work. For the sake of readability, the proofs of the new results are given in an Appendix.

\section{Preliminaries and brief state of art in knowledge dynamics}

\subsection{Notation}

We consider a propositional language $\mathcal{L}$ defined from a finite set of propositional variables $\mathcal{P}$ and the standard connectives.

An interpretation $\omega$ is a total function from $\mathcal{P}$ to $\{0,1\}$. The set of all interpretations is denoted by $\mathcal{W}$. An interpretation $\omega$ is a model of a formula $\varphi \in \mathcal{L}$ if and only if it makes it true in the usual truth functional way. $\bmod (\varphi)$ denotes the set of models of the formula $\varphi$, i.e., $\bmod (\varphi)=\{\omega \in \mathcal{W} \mid \omega \models \varphi\}$. When $M$ is a set of models we denote by $\varphi_{M}$ a formula such that $\bmod \left(\varphi_{M}\right)=M$. When $M$ is a singleton, say $M=\{\omega\}$, we use $\varphi_{\omega}$ instead of $\varphi_{\{\omega\}}$.

A belief base $K$ is a finite set of propositional formulae. In order to simplify the notations we identify the base $K$ with the formula $\varphi$ which is the conjunction of the formulae of $K^{5}$

A profile $\Psi$ is a non-empty multi-set (bag) of bases $\Psi=\left\{\varphi_{1}, \ldots, \varphi_{n}\right\}$ (hence different agents are allowed to exhibit identical bases), and represents a group of $n$ agents. For the sake of simplicity we will assume that each base in a profile is consistent. We denote by $\bigwedge \Psi$ the conjunction of bases of $\Psi=\left\{\varphi_{1}, \ldots, \varphi_{n}\right\}$, i.e., $\wedge \Psi=\varphi_{1} \wedge \ldots \wedge \varphi_{n}$. A profile $\Psi$ is said to be consistent if and only if $\wedge \Psi$ is consistent. The multi-set union ${ }^{6}$ is denoted by $\sqcup$, A profile $\Psi=\left\{\varphi_{1}, \ldots, \varphi_{n}\right\}$ is complete if all its bases are complete, i.e., $\forall \varphi_{i} \in \Psi, \varphi_{i}$ is complete.

A binary relation $\leq$ over $\mathcal{W}$ is said to be a preorder if it is transitive and reflexive. When the preorder $\leq$ is total (i.e., for any $\omega$ and $\omega^{\prime}$ in $\mathcal{W}, \omega \leq \omega^{\prime}$ or $\omega^{\prime} \leq \omega$ ) we call it a total preorder. The strict relation $<$ associated to a preorder $\leq$ is defined by $\omega<\omega^{\prime}$ iff $\omega \leq \omega^{\prime}$ and $\omega^{\prime} \not \leq \omega$.

\footnotetext{
${ }^{5}$ Some approaches are sensitive to syntactical representation. In that case it is important to distinguish between $K$ and the conjunction of its formulae (see e.g. [19]).

${ }^{6}$ This union takes into account the repetitions, thus, for instance, $\{a, b, b\} \sqcup\{a, b, c\}=$ $\{a, a, b, b, b, c\}$.
} 


\subsection{Revision}

Belief revision aims at changing the status of some beliefs in the base that are contradicted by a more reliable piece of information. Three main principles govern this revision operation. In priority order:

- First, the primacy of update principle: the new piece of information has to be accepted in the belief base after the revision. This is due to the hypothesis that the new piece of information is more reliable than the current beliefs of the agent. ${ }^{7}$

- Second, the principle of coherence: the new belief base after the revision should be a consistent belief base. Asking the beliefs of the agent to be consistent is a natural requirement if one wants the agent to conduct reasoning tasks from her belief base.

- Third, the principle of minimal change: the new belief base after the revision should be as close as possible from the current belief base of the agent. This important principle aims at ensuring that no unnecessary information (noise) is added to the beliefs of the agent during the revision process, and that no unnecessary information is lost during the process: information/beliefs are usually costly to obtain, we do not want to throw them away without any serious reason.

Alchourrón et al. [2] proposed some postulates in order to formalize these principles for belief revision. When one works with a finite propositional language the AGM postulates are equivalent to the following ones, proposed by Katsuno and Mendelzon:

Definition 1 [16] Let $\varphi$ and $\mu$ be two formulas denoting respectively the belief base of the agent, and a new piece of information. Then $\varphi \circ \mu$ is a formula representing the new belief base of the agent. An operator $\circ$ is an AGM belief revision operator if it satisfies the following properties:

(R1) $\varphi \circ \mu \vdash \mu$

(R2) If $\varphi \wedge \mu$ is consistent then $\varphi \circ \mu \equiv \varphi \wedge \mu$

(R3) If $\mu$ is consistent then $\varphi \circ \mu$ is consistent

(R4) If $\varphi_{1} \equiv \varphi_{2}$ and $\mu_{1} \equiv \mu_{2}$ then $\varphi_{1} \circ \mu_{1} \equiv \varphi_{2} \circ \mu_{2}$

(R5) $(\varphi \circ \mu) \wedge \phi \vdash \varphi \circ(\mu \wedge \phi)$

(R6) If $(\varphi \circ \mu) \wedge \phi$ is consistent then $\varphi \circ(\mu \wedge \phi) \vdash(\varphi \circ \mu) \wedge \phi$

(R1) states that the new piece of information must be believed after the revision. (R2) says that when there is no conflict between the new piece of information and the current beliefs of the agent, the revision is just the conjunction. (R3) says that revision always lead to a consistent belief base, unless the new piece of information is not consistent. (R4) is an irrelevance of syntax condition, it states that logically equivalent bases must lead to the same result. (R5) and (R6) give conditions on the revision by a conjunction.

\footnotetext{
${ }^{7}$ If this is not the case one should use a non-prioritized revision operator [13] or a merging operator (see Section 2.4).
} 
Alchourrón et al. also defined contraction operators, that aim to remove some piece of information from the beliefs of the agent. These contraction operators are closely related to revision operators, since each contraction operator can be used to define a revision operator, through the Levy identity and conversely each revision operator can be used to define a contraction operator through the Harper identity $[2,10]$. So one can study indifferently revision or contraction operators. So we focus on revision here.

Several representation theorems, that give constructive ways to define AGM revision/contraction operators, have been proposed, such as partial meet contraction/revision [2], epistemic entrenchments [10, 11], safe contraction [1], etc. In [16], Katsuno and Mendelzon give a representation theorem, showing that each revision operator corresponds to a faithful assignment, that associates to each base a plausibility preorder on interpretations (this idea can be traced back to Grove systems of spheres [12]).

Definition 2 A faithful assignment is a function mapping each base $\varphi$ to a pre-order $\leq_{\varphi}$ over interpretations such that:

1. If $\omega \models \varphi$ and $\omega^{\prime} \models \varphi$, then $\omega \simeq_{\varphi} \omega^{\prime}$

2. If $\omega \models \varphi$ and $\omega^{\prime} \not \models \varphi$, then $\omega<_{\varphi} \omega^{\prime}$

3. If $\varphi \equiv \varphi^{\prime}$, then $\leq_{\varphi}=\leq_{\varphi^{\prime}}$

Theorem 1 [16] An operator $\circ$ is an AGM revision operator (ie. it satisfies (R1)-(R6)) if and only if there exists a faithful assignment that maps each base $\varphi$ to a total pre-order $\leq_{\varphi} \operatorname{such}$ that $\bmod (\varphi \circ \mu)=\min \left(\bmod (\mu), \leq_{\varphi}\right)$.

Let us look now how to formalize the revision example given in the introduction:

Example 5 (Example 1 more formally) We represent the beliefs $\varphi$ of John about Linda with the propositional formula $f \wedge a$, where $f$ stands for activist in feminism and $a$ stands for academic position. For the sake of simplicity let us represent that bank teller, the new piece of information $\mu$, is represented by $\neg a$. Thus the problem is to revise $f \wedge a$ by $\neg a$. We can use the Dalal revision operator [7], using Hamming distance between interpretations $\left(d\left(\omega, \omega^{\prime}\right)\right.$ is the number of propositional variables in which $\omega$ and $\omega^{\prime}$ differs $)$ for defining a faithful assignment $\left(\varphi \mapsto \leq_{\varphi}\right)$ in the following way:

$$
\omega \leq_{\varphi} \omega^{\prime} \Leftrightarrow \min \left\{d\left(\omega, \omega^{\prime \prime}\right) \mid \omega^{\prime \prime} \in \bmod (\varphi)\right\} \leq \min \left\{d\left(\omega^{\prime}, \omega^{\prime \prime}\right) \mid \omega^{\prime \prime} \in \bmod (\varphi)\right\}
$$

It is easy to show that this assignment is a faithful assignment and then from Theo$\operatorname{rem} 1$, we have $\bmod \left(\varphi \circ_{D} \mu\right)=\min \left(\bmod (\mu), \leq_{\varphi}\right)=\min \left(\{(0,0),(1,0)\}, \leq_{\varphi}\right)=\{(1,0)\}$, that is $\varphi^{\circ}{ }_{D} \mu \equiv f \wedge \neg a$, which means that Linda is a feminist activist and a bank teller.

The kind of revision operators, defined by means of a distance, has been studied and characterized by Schlechta et al. [30] and Lehmann et al. [24]. 


\subsection{Update}

Whereas belief revision should be used to improve the (incorrect) beliefs of the agent by incorporating more reliable pieces of evidence, belief update operators aim at maintaining the belief base of the agent up-to-date, by allowing to modify the base according to a reported change in the world. This distinction between revision and update was made clear in [17], where Katsuno and Mendelzon proposed postulates for belief update.

Definition 3 [17] An operator $\diamond$ is a (partial) update operator if it satisfies the properties (U1)-(U8). It is a total update operator if it satisfies the properties (U1)(U5), (U8) and (U9).

(U1) $\varphi \diamond \mu \vdash \mu$

(U2) If $\varphi \vdash \mu$, then $\varphi \diamond \mu \equiv \varphi$

(U3) If $\varphi$ is consistent and $\mu$ is consistent then $\varphi \diamond \mu$ is consistent

(U4) If $\varphi_{1} \equiv \varphi_{2}$ and $\mu_{1} \equiv \mu_{2}$ then $\varphi_{1} \diamond \mu_{1} \equiv \varphi_{2} \diamond \mu_{2}$

(U5) $\quad(\varphi \diamond \mu) \wedge \phi \vdash \varphi \diamond(\mu \wedge \phi)$

(U6) If $\varphi \diamond \mu_{1} \vdash \mu_{2}$ and $\varphi \diamond \mu_{2} \vdash \mu_{1}$, then $\varphi \diamond \mu_{1} \equiv \varphi \diamond \mu_{2}$

(U7) If $\varphi$ is a complete formula, then $\left(\varphi \diamond \mu_{1}\right) \wedge\left(\varphi \diamond \mu_{2}\right) \vdash \varphi \diamond\left(\mu_{1} \vee \mu_{2}\right)$

(U8) $\left(\varphi_{1} \vee \varphi_{2}\right) \diamond \mu \equiv\left(\varphi_{1} \diamond \mu\right) \vee\left(\varphi_{2} \diamond \mu\right)$

(U9) If $\varphi$ is a complete formula and $(\varphi \diamond \mu) \wedge \phi \nvdash \perp$, then $\varphi \diamond(\mu \wedge \phi) \vdash(\varphi \diamond \mu) \wedge \phi$

Most of these postulates are close to the ones of revision. The main differences lie in postulate (U2) that is much weaker than (R2): conversely to revision, even if the new piece of information is consistent with the belief base, the result is generally not simply the conjunction. This illustrates the fact that revision can be seen as a selection process of the most plausible worlds of the current beliefs with respect to the new piece information, whereas update is a transition process: each world of the current beliefs have to be translated to the closest world allowed by the new piece of information. This world-by-world treatment is expressed by postulate (U8).

As for revision, there is a representation theorem in terms of faithful assignment.

Definition 4 A faithful assignment is a function mapping each interpretation $\omega$ to a pre-order $\leq_{\omega}$ over interpretations such that if $\omega \neq \omega^{\prime}$, then $\omega<_{\omega} \omega^{\prime}$.

One can easily check that this faithful assignment on interpretations is just a special case of the faithful assignment on bases defined in the previous section, when considering the complete bases corresponding to the interpretations.

Katsuno and Mendelzon give two representation theorems for update operators. The first representation theorem, that is the most commonly used, corresponds to partial pre-orders. This use of partial pre-order is one of the differences between belief revision and belief update (note nonetheless that postulates for belief revision can also be adapted to modelize assignments giving partial pre-orders [5]).

Theorem 2 [17] An update operator $\diamond$ satisfies (U1)-(U8) if and only if there exists a faithful assignment that maps each interpretation $\omega$ to a partial pre-order $\leq_{\omega}$ such that $\bmod (\varphi \diamond \mu)=\bigcup_{\omega \models \varphi} \min \left(\bmod (\mu), \leq_{\omega}\right)$. 
And there is also a second theorem corresponding to total pre-orders.

Theorem 3 [17] An update operator $\diamond$ satisfies (U1)-(U5), (U8) and (U9) if and only if there exists a faithful assignment that maps each interpretation $\omega$ to a total pre-order $\leq_{\omega} \operatorname{such}$ that $\bmod (\varphi \diamond \mu)=\bigcup_{\omega \models \varphi} \min \left(\bmod (\mu), \leq_{\omega}\right)$.

Notice that with the previous representation theorem is easy to see that postulates (U6) and (U7) follow from (U1)-(U5), (U8) and (U9). Thus, any total update operator is, in particular, a partial update operator.

This characterization of update is quite convincing, but some criticisms can be made that suggest that more elaborate update operators can be studied [15].

Let us illustrate update operators behavior by coming back to the introduction example:

Example 6 (Example 2 more formally) We consider the propositional variables, $b_{1}, b_{2}, \ldots, b_{5}$. Each variable $b_{i}$ stands for the book number $i$ is on the table. The current information $\varphi$ is $\left(b_{1} \wedge \neg b_{2} \wedge \neg b_{3} \wedge \neg b_{4} \wedge \neg b_{5}\right) \vee\left(\neg b_{1} \wedge \neg b_{2} \wedge b_{3} \wedge b_{4} \wedge\right.$ $b_{5}$ ) (That is either $B_{1}$ is the only book on the table or only $B_{3}, B_{4}$ and $B_{5}$ are on the table). The new piece of information, $\mu$, is $\left(b_{1} \wedge b_{2} \wedge b_{3} \wedge b_{4} \wedge b_{5}\right) \vee\left(\neg b_{1} \wedge \neg b_{2} \wedge\right.$ $\neg b_{3} \wedge \neg b_{4} \wedge \neg b_{5}$ ) (all the books are on the table or all the books are off the table).

We use Hamming distance in order to define the faithful assignment $\omega \mapsto \leq_{\omega}$, similarly to Example 5: $\omega_{1} \leq{ }_{w} \omega_{2} \quad \Leftrightarrow \quad d\left(\omega_{1}, \omega\right) \leq d\left(\omega_{2}, \omega\right)$

Since $\bmod (\varphi)=\{10000,00111\}$ and $\bmod (\mu)=\{00000,11111\}$, we have $\bmod \left(\varphi \diamond_{S} \mu\right)=$ $\bigcup_{\omega \models \varphi} \min \left(\bmod (\mu), \leq_{\omega}\right)=\min \left(\{00000,11111\}, \leq_{10000)}\right) \cup \min \left(\{00000,11111\}, \leq_{00111}\right)=$ $\{00000\} \cup\{11111\}$. That is, all the books are on the table or there are no books on the table. By contrast, the revision leads to the following situation: $\bmod \left(\varphi \circ_{D} \mu\right)=\min \left(\bmod (\mu), \leq_{\varphi}\right)=\min \left(\{00000,11111\}, \leq_{\varphi}\right)=\{00000\}$, that is there are no books on the table. But choosing $\{00000\}$ is strange since one does not know which of 00000 or 11111 the robot chosen.

Note that postulate (U8) captures the local nature of these operators. Actually, we have the following:

Proposition 1 Suppose $\diamond$ satisfies (U4) and (U8). If $\varphi \vdash \varphi^{\prime}$ then $\varphi \diamond \mu \vdash \varphi^{\prime} \diamond \mu$.

This property illustrates the locality property of update. As said in the introduction, revision operators, that work globally on the base do not satisfy this property.

\subsection{Merging}

Merging operators [3, 4, 25, 26, 29] should be used when one wants to combine several belief bases, or wants to take into account several pieces of information of same reliability.

Belief merging operators aim at aggregating several bases into a single one. The most basic case is when all the bases have the same strength/importance (see [8] for a discussion on prioritized merging). Often the aggregation has to obey a set of rules, that can be a translation of physical laws or of some knowledge about the result, that 
form the integrity constraints for the merging. Let us see the postulates for Integrity Constraints merging operators:

Definition 5 [20] Let $\Psi$ be a profile and $\mu$ be a formula encoding integrity constraints. Then $\triangle_{\mu}(\Psi)$ represents the merging of the profile $\Psi$ under the integrity constraints $\mu$. An operator $\Delta$ is an IC merging operator if it satisfies the following properties:

(IC0) $\Delta_{\mu}(\Psi) \vdash \mu$

(IC1) If $\mu$ is consistent, then $\triangle_{\mu}(\Psi)$ is consistent

(IC2) If $\bigwedge \Psi$ is consistent with $\mu$, then $\triangle_{\mu}(\Psi) \equiv \wedge \Psi \wedge \mu$

(IC3) If $\Psi_{1} \equiv \Psi_{2}$ and $\mu_{1} \equiv \mu_{2}$, then $\triangle_{\mu_{1}}\left(\Psi_{1}\right) \equiv \triangle_{\mu_{2}}\left(\Psi_{2}\right)$

(IC4) If $\varphi_{1} \vdash \mu$ and $\varphi_{2} \vdash \mu$, then $\triangle_{\mu}\left(\left\{\varphi_{1}, \varphi_{2}\right\}\right) \wedge \varphi_{1}$ is consistent if and only if $\triangle_{\mu}\left(\left\{\varphi_{1}, \varphi_{2}\right\}\right) \wedge \varphi_{2}$ is consistent

(IC5) $\triangle_{\mu}\left(\Psi_{1}\right) \wedge \triangle_{\mu}\left(\Psi_{2}\right) \vdash \triangle_{\mu}\left(\Psi_{1} \sqcup \Psi_{2}\right)$

(IC6) If $\triangle_{\mu}\left(\Psi_{1}\right) \wedge \triangle_{\mu}\left(\Psi_{2}\right)$ is consistent, then $\triangle_{\mu}\left(\Psi_{1} \sqcup \Psi_{2}\right) \vdash \triangle_{\mu}\left(\Psi_{1}\right) \wedge \triangle_{\mu}\left(\Psi_{2}\right)$

(IC7) $\triangle_{\mu_{1}}(\Psi) \wedge \mu_{2} \vdash \triangle_{\mu_{1} \wedge \mu_{2}}(\Psi)$

(IC8) If $\triangle_{\mu_{1}}(\Psi) \wedge \mu_{2}$ is consistent, then $\triangle_{\mu_{1} \wedge \mu_{2}}(\Psi) \vdash \triangle_{\mu_{1}}(\Psi)$

These postulates are quite close to the ones of revision. The ones that specifically talk about aggregation are (IC4), (IC5) and (IC6). (IC4) is a fairness postulate, that expresses the fact that all the bases have the same importance/weight, so when merging two such bases one can not give more importance to one of them. (IC5) and (IC6) talk about the result of the merging when we join two groups. (IC5) states that all that is common in the merging of the two groups must be selected if we join the two groups. And (IC6) strengthen this condition by asking that the merging obtained when we join the two groups have to be exactly what is commonly chosen by the two groups. These two postulates correspond to well known Pareto conditions (see conditions 5 and 6 of the syncretic assignment).

There is also a representation theorem for merging operators in terms of preorders on interpretations [20].

Definition 6 A syncretic assignment is a function mapping each profile $\Psi$ to a total pre-order $\leq_{\Psi}$ over interpretations such that:

1. If $\omega \models \Psi$ and $\omega^{\prime} \models \Psi$, then $\omega \simeq \Psi \omega^{\prime}$

2. If $\omega \models \Psi$ and $\omega^{\prime} \not \models \Psi$, then $\omega<_{\Psi} \omega^{\prime}$

3. If $\Psi_{1} \equiv \Psi_{2}$, then $\leq \Psi_{1}=\leq \Psi_{2}$

4. $\forall \omega \models \varphi \exists \omega^{\prime} \models \varphi^{\prime} \omega^{\prime} \leq_{\{\varphi\} \sqcup\left\{\varphi^{\prime}\right\}} \omega$

5. If $\omega \leq_{\Psi_{1}} \omega^{\prime}$ and $\omega \leq_{\Psi_{2}} \omega^{\prime}$, then $\omega \leq_{\Psi_{1} \sqcup \Psi_{2}} \omega^{\prime}$

6. If $\omega<_{\Psi_{1}} \omega^{\prime}$ and $\omega \leq_{\Psi_{2}} \omega^{\prime}$, then $\omega<_{\Psi_{1} \sqcup \Psi_{2}} \omega^{\prime}$

See [21] for a detailed explanation of these properties.

Theorem 4 [20] An operator $\triangle$ is an IC merging operator if and only if there exists a syncretic assignment that maps each profile $\Psi$ to a total pre-order $\leq_{\Psi}$ such that

$$
\bmod \left(\triangle_{\mu}(\Psi)\right)=\min \left(\bmod (\mu), \leq_{\Psi}\right)
$$


Let us illustrate the behavior of merging operator on the merging example of the introduction:

Example 7 (Example 3 more formally) Let us denote by $m$ the fact that dolphins are mammals. We represent the fact that dolphins are fishes by $\neg m$. Let us denote by $s$ the fact that dolphins have a sonar system, and by $d$ the fact that one can move without difficulty in the dark. Then we can translate the beliefs of the three girls. Margot believes that dolphins are mammals, so $\varphi_{M}=m$. Sofia believes that dolphins are fishes and that someone that have a sonar system can move without difficulty in the dark, so $\varphi_{S}=\neg m \wedge(s \rightarrow d)$. And Agathe believes that dolphins are mammals and that they have a sonar system, so $\varphi_{A}=m \wedge s$. So the profile is $\Psi=\left\{\varphi_{M}, \varphi_{S}, \varphi_{A}\right\}$, and there are no integrity constraints for the result, so $\mu=\top$. Now if one use the merging operator $\Delta^{\Sigma}$ [20], that is defined through the following syncretic assignment: $\omega \leq_{\Psi} \omega^{\prime} \Leftrightarrow \sum_{\varphi \in \Psi} d(\omega, \varphi) \leq \sum_{\varphi \in \Psi} d\left(\omega^{\prime}, \varphi\right)$, where $d(\omega, \varphi)=\min \left\{d\left(\omega, \omega^{\prime \prime}\right) \mid \omega^{\prime \prime} \models \varphi\right\}$ and $d$ is the Hamming distance then the resut obtained with some easy calculations is $\bmod \left(\triangle_{\mu}^{\Sigma}(\Psi)\right)=\min \left(\bmod (\mu), \leq_{\Psi}\right)=\min \left(\mathcal{W}, \leq_{\Psi}\right)=\{111\}$.

That means that the group beliefs are that dolphins are mammals, that have a sonar systems, and that can move without difficulty in the dark.

\subsection{On the links between revision, update and merging}

Let us see now what are the links between revision and update and between revision and merging. First, we begin with the links between revision and update.

Intuitively revision operators bring a minimal change to the base by selecting the most plausible models among the models of the new information. Whereas update operators bring a minimal change to each possible world (model) of the base in order to take into account the change described by the new infomation whatever the possible world. So, if we look closely to the representation theorems (Theorems 1, 2 and 3), we easily find the following result:

Theorem 5 If $\circ$ is a revision operator (i.e., it satisfies (R1)-(R6)), then the operator $\diamond$ defined by $\varphi \diamond \mu=\bigvee_{\omega \models \varphi} \varphi_{\omega} \circ \mu$ is an update operator that satisfies (U1)-(U9).

So this proposition states that update can be viewed as a kind of pointwise (model by model) revision. Now, we give some links between revision and merging. Intuitively revision operators select in a formula (the new evidence) the closest information to a ground information (the old base). And, identically, IC merging operators select in a formula (the integrity constraints) the closest information to a ground information (a profile of bases). So following this idea it is easy to make a correspondence between IC merging operators and belief revision operators:

Theorem 6 [20] If $\triangle$ is an IC merging operator (it satisfies (IC0)-(IC8)), then the operator $\circ$, defined as $\varphi \circ \mu=\triangle_{\mu}(\varphi)$, is an AGM revision operator (it satisfies (R1)(R6)).

See [20] for more links between belief revision and merging. 
Fig. 2 Revision-updatemerging-confluence

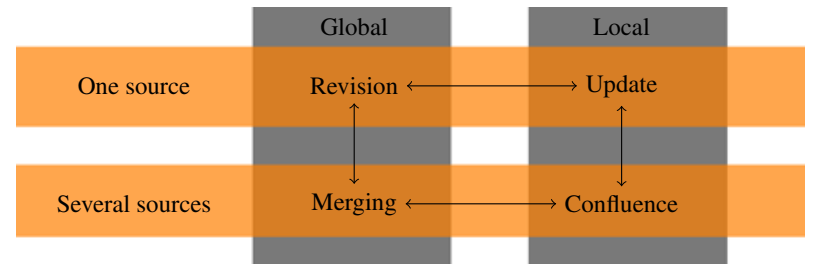

\section{Confluence operators}

As explained in the previous section, there are close connections between revision, update and merging. Update can be considered as a pointwise revision, and merging as a generalization of revision for several bases. So, as illustrated in Fig. 2, one can define confluence operators [22] that can be considered as a pointwise merging, and as a generalization of update for several bases.

These new operators are more cautious than merging operators. This suggest that they can be used as a first step of a conciliation process [18], that are a kind of abstract negotiation operators (see $[6,18,27,28,32])$, in order to find all the possible results of a conciliation process. See Section 5 for an illustration.

In order to illustrate the need for these new operators and also the difference of behaviour between merging and confluence we revisited Example 4 given in the introduction.

Example 8 Mary and Peter are planning to buy a car. Mary does not like a German car nor an expensive car. She likes small cars. Peter hesitates between a German, expensive but small car or a car which is not German, nor expensive and is a big car. Taking three propositional variables German_car, Expensive_car and Smal1_car in this order, Mary's desires are represented by $\bmod (A)=\{001\}$ and Peter's desires by $\bmod (B)=\{111,000\}$. Most of the merging operators ${ }^{8}$ give as solution a formula whose models are $\{001,000\}$. That is the same solution obtained when we suppose that Peter's desires are only a car which is not German nor expensive but a big car $\left(\bmod \left(B^{\prime}\right)=\{000\}\right)$. The confluence operators will take into account the disjunctive nature of Peter's desires in a better manner and they will incorporate also the interpretations that are a trade-off between 001 and 111. For instance, the worlds 011 and 101 will be also in the solution if one use the confluence operator $\diamond^{d_{H}, \operatorname{Gmax}}$ (defined in Section 4).

This kind of operators is particularly adequate when the base describes a situation that is not perfectly known, or that can evolve in the future. For instance Peter's desires can either be imperfectly known (he wants one of the two still possible situations but we do not know which one), or can evolve in the future (he will choose later between the two situations). In these situations the solutions proposed by confluence operators will be more adequate than the one proposed by merging operators. The solutions proposed by the confluence operators can be seen as all possible agreements in a conciliation (abstract negotiation) process.

$\overline{{ }^{8} \text { Such as } \triangle^{d_{H}, \Sigma} \text { and } \triangle^{d_{H}, G \max }}[20]$. 
Definition 7 An operator $\diamond$ is a confluence operator if it satisfies the following properties:

(UC0) $\diamond_{\mu}(\Psi) \vdash \mu$

(UC1) If $\mu$ is consistent then $\diamond_{\mu}(\Psi)$ is consistent.

(UC2) If $\Psi$ is complete, $\Psi$ is consistent and $\bigwedge \Psi \vdash \mu$, then $\diamond_{\mu}(\Psi) \equiv \bigwedge \Psi$

(UC3) If $\Psi_{1} \equiv \Psi_{2}$ and $\mu_{1} \equiv \mu_{2}$, then $\diamond_{\mu_{1}}\left(\Psi_{1}\right) \equiv \diamond_{\mu_{2}}\left(\Psi_{2}\right)$

(UC4) If $\varphi_{1}$ and $\varphi_{2}$ are complete formulae and $\varphi_{1} \vdash \mu, \varphi_{2} \vdash \mu$, then $\diamond_{\mu}\left(\left\{\varphi_{1}, \varphi_{2}\right\}\right) \wedge$ $\varphi_{1}$ is consistent if and only $\diamond_{\mu}\left(\left\{\varphi_{1}, \varphi_{2}\right\}\right) \wedge \varphi_{2}$ is consistent

(UC5) $\diamond_{\mu}\left(\Psi_{1}\right) \wedge \diamond_{\mu}\left(\Psi_{2}\right) \vdash \diamond_{\mu}\left(\Psi_{1} \sqcup \Psi_{2}\right)$

(UC6) If $\Psi_{1}$ and $\Psi_{2}$ are complete profiles and $\diamond_{\mu}\left(\Psi_{1}\right) \wedge \diamond_{\mu}\left(\Psi_{2}\right)$ is consistent, then $\diamond_{\mu}\left(\Psi_{1} \sqcup \Psi_{2}\right) \vdash \diamond_{\mu}\left(\Psi_{1}\right) \wedge \diamond_{\mu}\left(\Psi_{2}\right)$

(UC7) $\diamond_{\mu_{1}}(\Psi) \wedge \mu_{2} \vdash \diamond_{\mu_{1} \wedge \mu_{2}}(\Psi)$

(UC8) If $\Psi$ is a complete profile and if $\diamond_{\mu_{1}}(\Psi) \wedge \mu_{2}$ is consistent, then $\diamond_{\mu_{1} \wedge \mu_{2}}(\Psi) \vdash$ $\diamond_{\mu_{1}}(\Psi) \wedge \mu_{2}$

(UC9) $\diamond_{\mu}\left(\Psi \sqcup\left\{\varphi \vee \varphi^{\prime}\right\}\right) \equiv \diamond_{\mu}(\Psi \sqcup\{\varphi\}) \vee \diamond_{\mu}\left(\Psi \sqcup\left\{\varphi^{\prime}\right\}\right)$

Some of the (UC) postulates are exactly the same as (IC) ones, just like some (U) postulates for update are exactly the same as $(\mathrm{R})$ ones for revision.

In fact, (UC0), (UC3), (UC5) and (UC7) are exactly the same as the corresponding (IC) postulates. So the specificity of confluence operators lies in postulates (UC1), (UC2), (UC6), (UC8) and (UC9). (UC1) tells us about consistency of the result. (UC2), (UC4), (UC6) and (UC8) are close to the corresponding (IC) postulates, but hold for complete profiles only. The present formulation of (UC2) is quite similar to formulation of (U2) for update. Note that in the case of a complete profile the hypothesis of (UC2) is equivalent to ask coherence with the constraints, i.e., the hypothesis of (IC2). Postulates (UC8) and (UC9) are the main difference with merging postulates, and correspond also to the main difference between revision and KM update operators. (UC9) is the most important postulate, that defines confluence operators as pointwise agregation, just like (U8) defines update operators as pointwise revision. This will be expressed more formally in the next subsection (see Lemma 1).

\subsection{Representation theorem for confluence operators}

In order to state the representation theorem for confluence operators, we first have to be able to "localize" the problem. For update this is done by looking to each model of the base, instead of looking at the base (set of models) as a whole. So for "localizing" the aggregation process, we have to find what is the local view of a profile. That is what we call a state.

Definition 8 A multi-set of interpretations will be called a state. We use the letter $e$, possibly with subscripts, for denoting states. If $\Psi=\left\{\varphi_{1}, \ldots, \varphi_{n}\right\}$ is a profile and $e=\left\{\omega_{1}, \ldots, \omega_{n}\right\}$ is a state such that $\omega_{i} \models \varphi_{i}$ for each $i$, we say that $e$ is a state of the profile $\Psi$, or that the state $e$ models the profile $\Psi$, that will be denoted by $e \models \Psi$. If $e=\left\{\omega_{1}, \ldots, \omega_{n}\right\}$ is a state, we define the profile $\Psi_{e}$ by putting $\Psi_{e}=\left\{\varphi_{\omega_{1}}, \ldots, \varphi_{\omega_{n}}\right\}$. 
State is an interesting notion. If we consider each base as the current point of view (goals) of the corresponding agent (that can be possibly strengthened in the future) then states are all possible conciliation (negotiation) starting points.

States are the points of interest for confluence operators (like interpretations are for update), as stated in the following important Lemma:

Lemma 1 If $\diamond$ satisfies (UC3) and (UC9) then $\diamond$ satisfies the following

$$
\diamond_{\mu}(\Psi) \equiv \bigvee_{e \models \Psi} \diamond_{\mu}\left(\Psi_{e}\right)
$$

Defining profile entailment by putting $\Psi \vdash \Psi^{\prime}$ iff every state of $\Psi$ is a state of $\Psi^{\prime}$, the previous Lemma has as a corollary the following result which is capturing exactly the local nature of confluence operators:

Corollary 1 If $\diamond$ is a confluence operator then it is monotonic in the profiles, that means that if $\Psi \vdash \Psi^{\prime}$ then $\diamond_{\mu}(\Psi) \vdash \diamond_{\mu}\left(\Psi^{\prime}\right)$.

This monotony property, that is not true in the case of merging operators, shows one of the big differences between merging and confluence operators. Remark that there is a corresponding monotony property for update (see Proposition 1).

Like revision's faithful assignments that have to be "localized" to interpretations for update, merging's syncretic assignments have to be localized to states for confluence.

Definition 9 A distributed assignment is a function mapping each state $e$ to a total pre-order $\leq_{e}$ over interpretations such that:

1. $\omega<\{\omega, \ldots, \omega\} \omega^{\prime}$ if $\omega^{\prime} \neq \omega$

2. $\omega \simeq\left\{\omega, \omega^{\prime}\right\} \omega^{\prime}$

3. If $\omega \leq_{e_{1}} \omega^{\prime}$ and $\omega \leq_{e_{2}} \omega^{\prime}$, then $\omega \leq_{e_{1} \sqcup e_{2}} \omega^{\prime}$

4. If $\omega<_{e_{1}} \omega^{\prime}$ and $\omega \leq_{e_{2}} \omega^{\prime}$, then $\omega<_{e_{1} \sqcup e_{2}} \omega^{\prime}$

The conditions 3 and 4 are similar to Pareto conditions in Social Choice Theory they express the deep relations between the "individual" preorders $\leq_{e_{i}}$ and the "social" preorder $\leq_{e_{1} \sqcup e_{2}}$.

Now we can state the main result of this paper, that is the representation theorem for confluence operators.

Theorem 7 An operator $\diamond$ is a confluence operator if and only if there exists a distributed assignment that maps each state e to a total pre-order $\leq_{e}$ such that

$$
\bmod \left(\diamond_{\mu}(\Psi)\right)=\bigcup_{e \models \Psi} \min \left(\bmod (\mu), \leq_{e}\right)
$$

\subsection{Confluence vs update and merging}

So now we are able to state the proposition that shows that update is a special case of confluence, just as revision is a special case of merging. 
Theorem 8 If $\diamond$ is a confluence operator (i.e., it satisfies (UC0)-(UC9)), then the operator $\diamond$, defined as $\varphi \diamond \mu=\diamond_{\mu}(\varphi)$, is an update operator (i.e., it satisfies (U1)(U9)).

Concerning merging operators, one can easily see that the restriction of a syncretic assignment to a complete profile is a distributed assignment. From that we obtain the following result (the one corresponding to Theorem 5):

Theorem 9 If $\triangle$ is an IC merging operator (i.e., it satisfies (IC0)-(IC8)) then the operator $\diamond$ defined by

$$
\diamond_{\mu}(\Psi)=\bigvee_{e \models \Psi} \triangle_{\mu}\left(\Psi_{e}\right)
$$

is a confluence operator (i.e., it satisfies (UC0)-(UC9)).

As a corollary of the representation theorem we obtain the following

Corollary 2 If $\diamond$ is a confluence operator then the following property holds:

If $\wedge \Psi \vdash \mu$ and $\Psi$ is consistent then $\wedge \Psi \wedge \mu \vdash \diamond_{\mu}(\Psi)$

But unlike merging operators, we don't have generally $\diamond_{\mu}(\Psi) \vdash \wedge \Psi \wedge \mu$.

Note that this "half of (IC2)" property is similar to the "half of (R2)" satisfied by update operators.

This corollary is interesting since it underlines an important difference between merging and confluence operators. If all the bases agree (i.e., if their conjunction is consistent), then a merging operator gives as result exactly the conjunction, whereas a confluence operator will give this conjunction plus additional results. This is useful if the bases represent uncertain information about the agent's current or future state of mind.

\section{General construction of confluence operators based on distances}

In this section, a general construction of confluence operators based on distances on worlds and aggregation functions is provided. After that the behaviour of confluence operators is illustrated on an example.

Definition 10 A pseudo-distance $e^{9}$ between interpretations is a total function $d: \mathcal{W} \times$ $\mathcal{W} \mapsto \mathbb{R}^{+}$s.t. for any $\omega, \omega^{\prime} \in \mathcal{W}$ :

- $d\left(\omega, \omega^{\prime}\right)=d\left(\omega^{\prime}, \omega\right)$, and

- $d\left(\omega, \omega^{\prime}\right)=0$ if and only if $\omega=\omega^{\prime}$.

\footnotetext{
${ }^{9}$ Notice that we don't ask for the triangle inequality in this definition. Actually, a distance is pseudodistance satisfying the triangle inequality.
} 
A widely used distance between interpretations is the Dalal distance [7], denoted $d_{H}$, that is the Hamming distance between interpretations (the number of propositional variables on which the two interpretations differ).

Definition 11 An (symmetric) aggregation function $f$ is a total function associating a nonnegative real number to every finite tuple of nonnegative real numbers such that for any $x_{1}, \ldots, x_{n}, x, y \in \mathbb{R}^{+}$:

- if $x \leq y$, then $f\left(x_{1}, \ldots, x, \ldots, x_{n}\right) \leq f\left(x_{1}, \ldots, y, \ldots, x_{n}\right) \quad$ (non-decreasingness)

$-f\left(x_{1}, \ldots, x_{n}\right)=0$ if and only if $x_{1}=\ldots=x_{n}=0$

(minimality)

$-f(x)=x$

(identity)

- For any permutation $\sigma, f\left(x_{1}, \ldots, x_{n}\right)=f\left(\sigma\left(x_{1}, \ldots, x_{n}\right)\right)$ (symmetry) [20].

Well known aggregation functions are for instance max, sum, or leximax $(\mathrm{Gmax})^{10}$

We are going to define operators using two parameters: a pseudo-distance and an aggregation function. Under certain very natural conditions on the aggregation functions these operators will be confluence operators as we will see below.

Definition 12 (Distance-based operators) Let $d$ be a pseudo-distance between interpretations and $f$ be an aggregation function. The result $\diamond_{\mu}^{d, f}(\Psi)$, of changing the profile $\Psi$ given the integrity constraints $\mu$ is defined by:

$$
\bmod \left(\diamond_{\mu}^{d, f}(\Psi)\right)=\bigcup_{e \models \Psi} \min \left(\bmod (\mu), \leq_{e}\right)
$$

where the pre-order $\leq_{e}$ on $\mathcal{W}$ induced by $e$ is defined by:

- $\quad \omega \leq_{e} \omega^{\prime}$ if and only if $d(\omega, e) \leq d\left(\omega^{\prime}, e\right)$, where

$-\quad d(\omega, e)=f\left(d\left(\omega, \omega_{1}\right) \ldots, d\left(\omega, \omega_{n}\right)\right)$ with $e=\left\{\omega_{1}, \ldots, \omega_{n}\right\}$.

Let us define two additional properties for aggregation functions [19]:

- If $f\left(x_{1}, \ldots, x_{n}\right) \leq f\left(y_{1}, \ldots, y_{n}\right)$, then

$f\left(x_{1}, \ldots, x_{n}, z\right) \leq f\left(y_{1}, \ldots, y_{n}, z\right)$ (composition)

- If $f\left(x_{1}, \ldots, x_{n}, z\right) \leq f\left(y_{1}, \ldots, y_{n}, z\right)$, then

$f\left(x_{1}, \ldots, x_{n}\right) \leq f\left(y_{1}, \ldots, y_{n}\right)$ (decomposition)

These properties are very natural. Composition says that if we add a same value to two different profiles, then it does not change their (aggregated) value order. Decomposition is the opposite property stating that removing a same value from two different profiles does not change their value order.

Theorem 10 Let $d$ be a pseudo-distance and $f$ be an aggregation function. If the aggregation function $f$ satisfies composition and decomposition, then the distancebased operator $\diamond^{d, f}$ is a confluence operator (i.e., it satisfies (UC0)-(UC9)).

\footnotetext{
${ }^{10}$ Leximax (Gmax) is usually defined using lexicographic sequences, but it can be easily represented by reals to fit the above definition (see e.g. [19]).
} 
The previous theorem gives sufficient conditions on aggregation function to obtain a distance-based confluence operator. In order to establish a full characterization of properties on aggregation functions determining distance-based confluence operators we need to circumscribe a class of numbers definable with a distance. This is the purpose of the next definition.

Definition 13 Let $d$ be a pseudo-distance. Let $\vec{x}, \vec{y}$ be two vectors of nonnegative real numbers of the same size (say $n$ ) and $z$ a nonnegative real number. We say that the triple $\vec{x}, \vec{y}, z$ is $d$-realizable iff there are worlds $\omega, \omega^{\prime}, \omega^{\prime \prime}, \omega_{1}, \ldots, \omega_{n}$ such that:

$$
\begin{aligned}
\vec{x} & =\left(d\left(\omega, \omega_{1}\right), \ldots, d\left(\omega, \omega_{n}\right)\right) \\
\vec{y} & =\left(d\left(\omega^{\prime}, \omega_{1}\right), \ldots, d\left(\omega^{\prime}, \omega_{n}\right)\right) \\
z & =d\left(\omega, \omega^{\prime \prime}\right)=d\left(\omega^{\prime}, \omega^{\prime \prime}\right)
\end{aligned}
$$

Definition 14 Let $d$ be a pseudo-distance. An aggregation function $f$ satisfies $d$ composition (respectively $d$-decomposition) iff $f$ satisfies composition (respectively decomposition) for all $d$-realizable triples.

Let us now state the result on the links between the properties of the confluence operator and the ones of the aggregation function.

Theorem 11 Let $d$ be a pseudo-distance and $f$ be an aggregation function. Then the distance-based operator $\diamond^{d, f}$ is a confluence operator (i.e., it satisfies (UC0)-(UC9)) if and only if the aggregation function $f$ satisfies $d$-composition and d-decomposition.

It is easy to check that the functions $\Sigma$ and Gmax are aggregations functions satisfying composition and decomposition. Therefore, as a corollary of Theorem 10, we have the following result:

Theorem 12 Let $d$ be any distance, $\diamond^{d, \Sigma}$ and $\diamond^{d, G m a x}$ are confluence operators (i.e., they satisfy (UC0)-(UC9)).

The following example illustrates the behavior of the operators built using the previous definition.

Example 9 Let us consider a profile $\Psi=\left\{\varphi_{1}, \varphi_{2}, \varphi_{3}, \varphi_{4}\right\}$ and an integrity constraint $\mu$ defined on a propositional language built over four symbols, as follows: $\bmod (\mu)=$ $\mathcal{W} \backslash\{0110,1010,1100,1110\}, \bmod \left(\varphi_{1}\right)=\bmod \left(\varphi_{2}\right)=\{1111,1110\}, \bmod \left(\varphi_{3}\right)=\{0000\}$, and $\bmod \left(\varphi_{4}\right)=\{1110,0110\}$.

The computations for the operators $\diamond^{d_{H}, \Sigma}$ and $\diamond^{d_{H}, G m a x}$, that use respectively the sum and the leximax as aggregation function, and that both use the Hamming distance $d_{H}$ are reported in Table 1 . The shadowed lines correspond to the interpretations rejected by the integrity constraints. Thus the result has to be taken among 


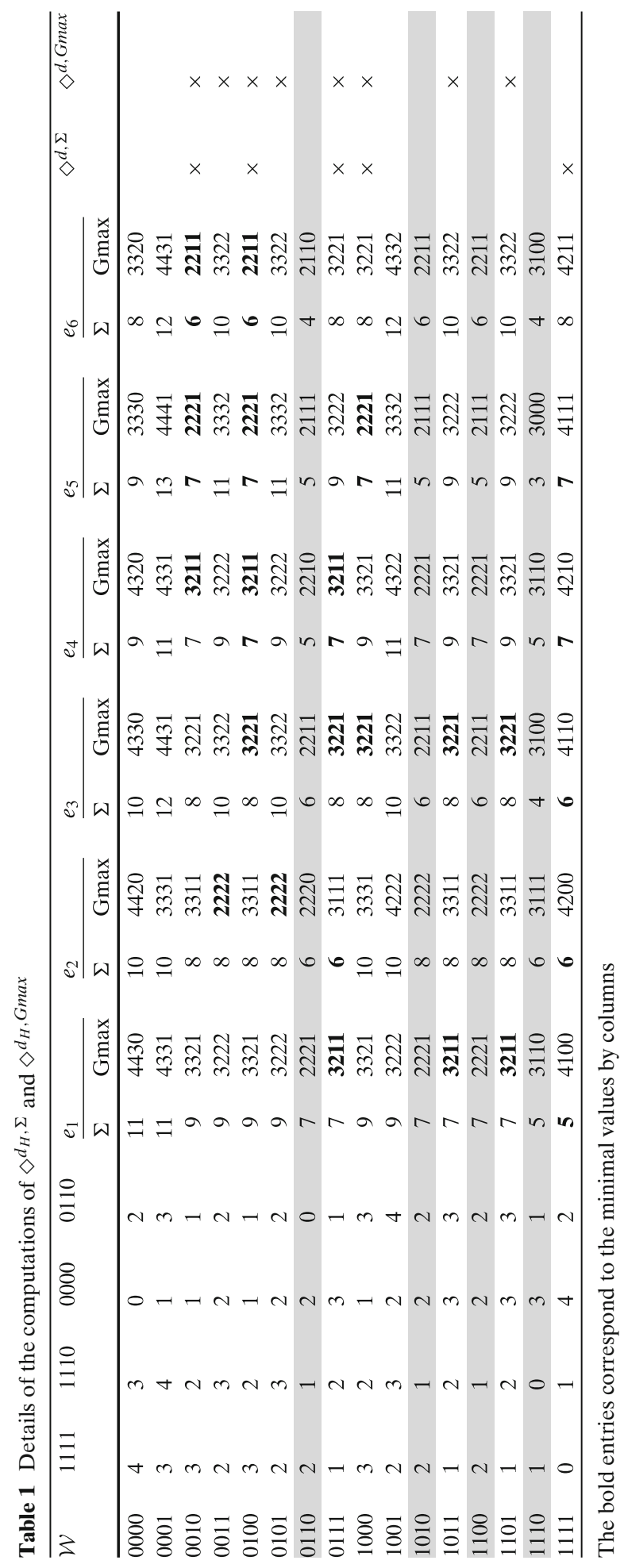


the interpretations that are not shadowed. The states that model the profile are the following ones:

$$
\begin{array}{ll}
e_{1}=\{1111,1111,0000,1110\}, & e_{2}=\{1111,1111,0000,0110\}, \\
e_{3}=\{1111,1110,0000,1110\}, & e_{4}=\{1110,1111,0000,0110\}, \\
e_{5}=\{1110,1110,0000,1110\}, & e_{6}=\{1110,1110,0000,0110\} .
\end{array}
$$

For each interpretation, the table gives the distance between the interpretation and the four interpretations that appear in the different states (first four columns), then the distance between the interpretation and each state for the $\Sigma$ aggregation function, and for the Gmax one. So one can then look at the best interpretations for each state (i.e., the ones with minimal aggregated value). So there is a cross in the last column if this interpretation is selected (by at least one state) for the $\diamond^{d_{H}, G m a x}$ operator, and similarly there is a cross in the next-to-the-last column if the interpretation is selected by $\diamond^{d_{H}, \Sigma}$.

So for instance for $\mho_{\mu}^{d, \Sigma}(\Psi), e_{1}$ selects the interpretation $1111, e_{2}$ selects 0111 and 1111 , etc. So, taking the union of the interpretations selected by each state, gives $\bmod \left(\diamond_{\mu}^{d, \Sigma}(\Psi)\right)=\{0010,0100,0111,1000,1111\}$.

Similarly we obtain $\bmod \left(\diamond_{\mu}^{d, G \max }(\Psi)\right)=\{0100,0011,0010, \quad 0101,0111,1000$, $1011,1101\}$.

\section{Conclusion and perspectives}

In this paper, we investigated the links between revision, update and merging operators, and illustrated the lack of one operator in this picture, we introduced confluence operators, that are to merging what update operators are to revision (or, symmetrically confluence operators can be considered to be to update, what merging operators are to revision). We proposed a set of postulates for characterizing these confluence operators and a representation theorem in terms of pre-orders on interpretations. We showed that we obtained the expected links with update and merging operators and we provided a way to build concrete confluence operators.

A first perspective of this work comes from the remark that update operators are mostly known using partial pre-orders, whereas our confluence operators use total pre-orders. So it could be interesting to see if a partial version (i.e., where the corresponding faithful assignment works with partial pre-orders instead of total ones) of confluence operators can be defined.

As another perspective we finally want to discuss the possible use of confluence operators in a process of conciliation (abstract negotiation) [18] between agents having different points of view (goals or desires).

We think that the output of a confluence operator can be considered as all the possible outcomes in a conciliation process between agents. In such a process the number of outcomes is usually big. Thus, in order to reduce the research space of solutions in a conciliation process, one would like a rational method for selecting only some of these possible outcomes. We provide such a method in this section. We think that the study of such methods is an interesting topic of its own, so the proposed method in this section has just to be considered as an illustration and a starting point for future research. 
In order to motivate the definition of our selection method let us come back to Example 9. The result of the confluence operator $\left(\diamond^{d, \Sigma}\right.$ and $\diamond^{d, G m a x}$ in Example 9) is the set of interpretations that are selected by at least one state. No distinction is made on the number of times an interpretation is selected. Let us look at Table 1, concerning the two interpretations 0011,0100 selected as outcome of $\diamond^{d, G m a x}$ : whereas 0011 is selected by only one state $\left(e_{2}\right)$, the interpretation 0100 is selected by four states $\left(e_{3}, e_{4}, e_{5}, e_{6}\right)$ out of six. So this outcome should be considered as more plausible than 0011 .

Hence the selection method idea is to consider how often each outcomes are selected by the different states. Let us see this on Table 2.

On this table we can see that for $\diamond^{d, \Sigma}$ the interpretation 1111 is selected by 5 states out of 6 . And for $\diamond^{d, G m a x}$ the interpretation 0100 is selected by 4 states out of 6 .

To justify the use of these numbers, one can consider our first interpretation of confluence operators: Suppose that the belief bases provided by the agents are an uncertain approximation of the point of view of each agent. Each agent will finally defend one position (complete formula), but we do not know which one. The only think we know is that it will be one of the positions allowed by the corresponding belief base. In this case we can use a principle of insufficient reason, and suppose that there is an equiprobability on the choice of the final position defended by the agent. So this means that the probability of each state to finally occur is also an equiprobability. In particular, the more states select a given interpretations as possible outcome, the more plausible is this outcome.

Let us define this method formally:

Definition 15 Let $\diamond$ be a confluence operator. Let $\Psi$ be a profile and $\omega$ be an interpretation, define

$$
m s_{\diamond_{\mu}(\Psi)}(\omega)=\left|\left\{e \in E \mid \omega \models \diamond_{\mu}\left(\Psi_{e}\right)\right\}\right|
$$

Then the majoritarian confluence operator $\oplus$ is defined as:

$$
\bmod \left(\oplus_{\mu}(E)\right)=\operatorname{argmax}_{\omega \models \mu} m s_{\diamond_{\mu}(\Psi)}(\omega)
$$

Clearly with this definition we obtain on Example $9 \bmod \left(\oplus_{\mu}^{d, \Sigma}(\Psi)\right)=\{1111\}$ and $\bmod \left(\oplus_{\mu}^{d, G \max }(\Psi)\right)=\{0100\}$.

Finally, we obtain the following result:

Theorem 13 Let $\diamond$ be a confluence operator and $\diamond$ the corresponding majoritarian confluence operator. The operator $\oplus$ satisfies (UC1)-(UC8). It does not satisfy (UC9).

These operators are interesting since they allow to obtain much more precise results than the corresponding confluence operators, but this has to be paid by the lost of UC9. Considering these operators, or related ones, based on a selection of a subset of the interpretations selected by the confluence operators, as conciliation operators seem promising. We plan to investigate more this issue, and the links with other works on abstract negotiation [6, 18, 27, 28, 32]. 


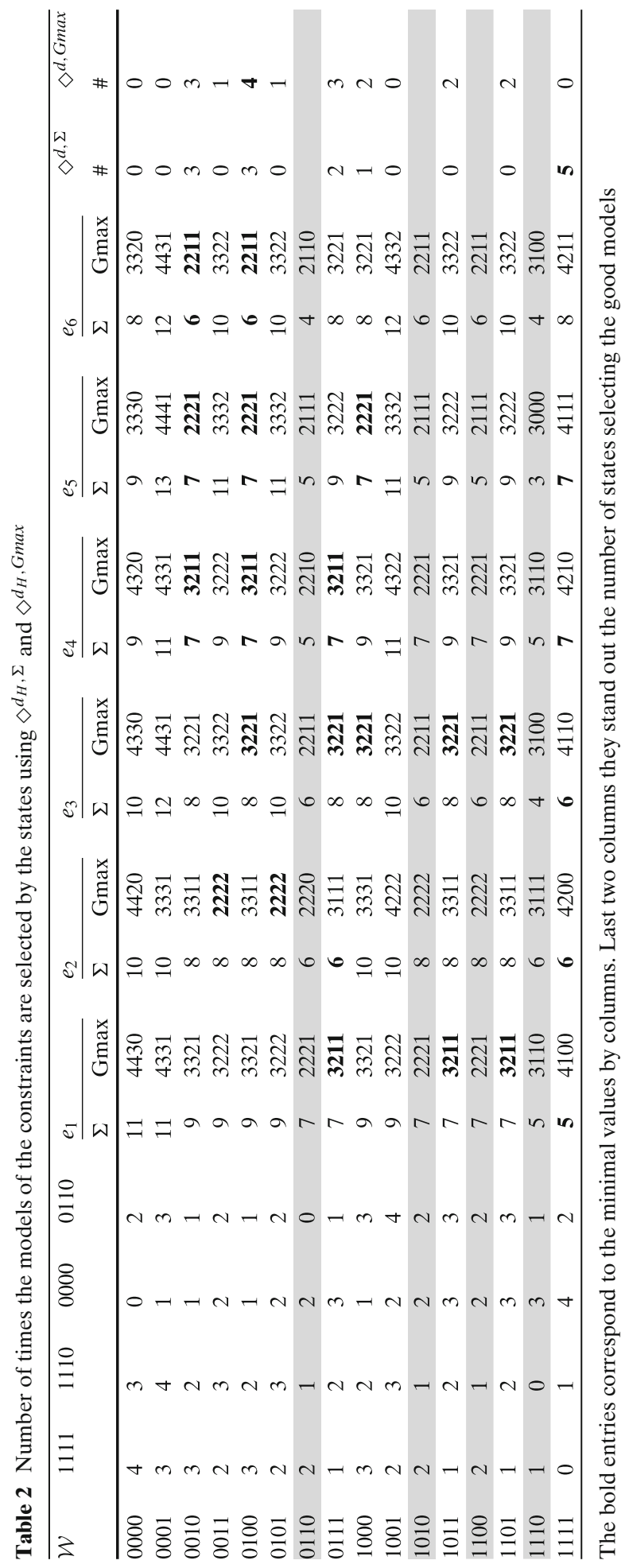


Acknowledgements The idea of this paper comes from discussions in the 2005 and 2007 Dagstuhl seminars (\#5321 and \#7351) "Belief Change in Rational Agents". The authors would like to thank the Schloss Dagstuhl institution, and the participants of the seminars, especially Andreas Herzig for his interesting question: "If merging can be seen as a generalization of revision, what is the generalization of update?". Here is an answer!

The authors would like to thank the anonymous reviewers for their helpful comments. The second author wishes to thank the Université d'Artois for a visiting position.

\section{Appendix: Proofs}

Proof of Lemma 1 We proceed by induction on $k$, the number of states satisfying the profile. Suppose $\Psi=\left\{\varphi_{1}, \ldots, \varphi_{n}\right\}$. If the number of states of $\Psi, k$, is equal to 1 , then there are $\omega_{1}, \ldots, \omega_{n}, \omega_{n+1}$ such that $\varphi_{i} \equiv \varphi_{\omega_{i}}$, for $i=1, \ldots, n$. Then by (UC3),

$$
\diamond_{\mu}(\Psi)=\diamond_{\mu}\left(\left\{\varphi_{\omega_{1}}, \ldots, \varphi_{\omega_{n}}\right\}\right)=\bigvee_{e \models \Psi} \diamond_{\mu}\left(\Psi_{e}\right)
$$

The last equality holds because the unique state satisfying $\Psi$ is $\left(\omega_{1}, \ldots, \omega_{n}\right)$. Now suppose the claim holds for all the profiles having at most $k$ states and suppose that $\Psi$ has $k+1$ states. Necessarily there is a $\varphi_{i}$ in $\Psi$ such that $\left|\bmod \left(\varphi_{i}\right)\right| \geq 2$. Without loss of generality we can suppose $i=n$. Then there are formulas $\varphi, \varphi^{\prime}$ such that $\varphi_{n} \equiv \varphi \vee \varphi^{\prime}$ and $\bmod (\varphi) \cap \bmod \left(\varphi^{\prime}\right)=\varnothing$. Put $\Psi^{1}=\left\{\varphi_{1}, \ldots, \varphi_{n-1}, \varphi\right\}$ and $\Psi^{2}=\left\{\varphi_{1}, \ldots, \varphi_{n-1}, \varphi^{\prime}\right\}$. Clearly the number of states of $\Psi^{i}$ is less or equal to $k$ for $i=1,2$. Then, by (UC9),

$$
\diamond_{\mu}(\Psi)=\diamond_{\mu}\left(\Psi^{1}\right) \vee \diamond_{\mu}\left(\Psi^{2}\right)
$$

By induction hypothesis,

$$
\diamond_{\mu}\left(\Psi^{1}\right) \vee \diamond_{\mu}\left(\Psi^{2}\right)=\bigvee_{e \models \Psi^{1}} \diamond_{\mu}\left(\Psi_{e}^{1}\right) \vee \bigvee_{e \models \Psi 2} \diamond_{\mu}\left(\Psi_{e}^{2}\right)
$$

But note that $\{e: e \models \Psi\}=\left\{e: e \models \Psi^{1}\right\} \cup\left\{e: e \models \Psi^{2}\right\}$, thus we have

$$
\bigvee_{e \models \Psi^{1}} \diamond_{\mu}\left(\Psi_{e}^{1}\right) \vee \bigvee_{e \models \Psi 2} \diamond_{\mu}\left(\Psi_{e}^{2}\right)=\bigvee_{e \models \Psi} \diamond_{\mu}\left(\Psi_{e}\right)
$$

and by transitivity

$$
\diamond_{\mu}(\Psi)=\bigvee_{e \models \Psi} \diamond_{\mu}\left(\Psi_{e}\right)
$$

\section{Proof of Theorem 7}

(Only if part) Let $\diamond$ be a confluence operator. Let us define a distributed assignment as follows: for each state $e$ we define a total pre-order $\leq_{e}$ by putting $\forall \omega, \omega^{\prime} \in \mathcal{W} \omega \leq_{e} \omega^{\prime}$ if and only if $\omega \models \diamond_{\varphi_{\left\{\omega, \omega^{\prime}\right\}}}\left(\Psi_{e}\right)$. Note that, in virtue of (UC3), the definition of $\leq_{e}$ does not depends on the (syntactic) choice of $\varphi_{\left\{\omega, \omega^{\prime}\right\}}$.

First we show that $\leq_{e}$ is a total pre-order:

Totality $\forall \omega, \omega^{\prime} \in \mathcal{W}$, since $\varphi_{\left\{\omega, \omega^{\prime}\right\}}$ is consistent, and $\Psi_{e}$ is consistent, from (UC1) part (a), we have $\diamond_{\varphi_{\left\{\omega, \omega^{\prime}\right\}}}\left(\Psi_{e}\right) \nvdash \perp$ and from (UC0) $\diamond_{\varphi_{\left\{\omega, \omega^{\prime}\right\}}}\left(\Psi_{e}\right) \vdash \varphi_{\left\{\omega, \omega^{\prime}\right\}}$, so either 
$\omega \in \bmod \left(\diamond_{\varphi_{\left\{\omega, \omega^{\prime}\right\}}}\left(\Psi_{e}\right)\right)$ that means by definition $\omega \leq_{e} \omega^{\prime}$, or $\omega^{\prime} \in \bmod \left(\diamond_{\varphi_{\left\{\omega, \omega^{\prime}\right\}}}\left(\Psi_{e}\right)\right)$, that means $\omega^{\prime} \leq_{e} \omega$.

Reflexivity From (UC0) and (UC1) part (a) we have that $\diamond_{\varphi_{\{\omega\}}}\left(\Psi_{e}\right)=\varphi_{\{\omega\}}$. So $\omega \leq_{e} \omega$.

Transitivity Assume that $\omega \leq_{e} \omega^{\prime}$ and $\omega^{\prime} \leq_{e} \omega^{\prime \prime}$ and suppose towards a contradiction that $\omega \bigsqcup_{e} \omega^{\prime \prime}$. As $\leq_{e}$ is total, this means $\omega^{\prime \prime}<_{e} \omega$, so by definition $\diamond_{\varphi_{\left\{\omega, \omega^{\prime \prime}\right\}}}\left(\Psi_{e}\right) \equiv \varphi_{\left\{\omega^{\prime \prime}\right\}}$. By (UC7) we find that $\diamond_{\varphi_{\left\{\omega, \omega^{\prime}, \omega^{\prime \prime}\right\}}}\left(\Psi_{e}\right) \wedge \varphi_{\left\{\omega, \omega^{\prime \prime}\right\}} \vdash \diamond_{\varphi_{\left\{\omega, \omega^{\prime \prime}\right\}}}\left(\Psi_{e}\right)$. We consider two cases:

Case $1 \diamond_{\varphi_{\left\{\omega, \omega^{\prime}, \omega^{\prime \prime}\right\}}}\left(\Psi_{e}\right) \wedge \varphi_{\left\{\omega, \omega^{\prime \prime}\right\}}$ is consistent. Then, by (UC7), (UC8) and the hypothesis, $\diamond_{\varphi_{\left\{\omega, \omega^{\prime}, \omega^{\prime \prime}\right\}}}\left(\Psi_{e}\right) \wedge \varphi_{\left\{\omega, \omega^{\prime \prime}\right\}} \equiv \varphi_{\left\{\omega^{\prime \prime}\right\}}$. So we have $\omega \not \models \diamond_{\varphi_{\left\{\omega, \omega^{\prime}, \omega^{\prime \prime}\right\}}}\left(\Psi_{e}\right)$. But by (UC1) part (a) we have $\diamond_{\varphi_{\left\{\omega, \omega^{\prime}, \omega^{\prime \prime}\right\}}}\left(\Psi_{e}\right) \nvdash \perp$, and by (UC0) we know that either $\bmod \left(\diamond_{\varphi_{\left\{\omega, \omega^{\prime}, \omega^{\prime \prime}\right\}}}\left(\Psi_{e}\right)\right)=\left\{\omega^{\prime}, \omega^{\prime \prime}\right\}$ or $\bmod \left(\diamond_{\varphi_{\left\{\omega, \omega^{\prime}, \omega^{\prime \prime}\right\}}}\left(\Psi_{e}\right)\right)=\left\{\omega^{\prime \prime}\right\}$. In the first case by (UC7) and (UC8) we conclude that $\diamond_{\varphi_{\left\{\omega, \omega^{\prime}, \omega^{\prime \prime}\right\}}}\left(\Psi_{e}\right) \wedge \varphi_{\left\{\omega, \omega^{\prime}\right\}} \equiv \diamond_{\varphi_{\left\{\omega, \omega^{\prime}\right\}}}\left(\Psi_{e}\right)$ and so $\omega \not \models \diamond_{\varphi_{\left\{\omega, \omega^{\prime}\right\}}}\left(\Psi_{e}\right)$. Contradiction. In the second case by (UC7) and (UC8) we obtain $\diamond_{\varphi_{\left\{\omega, \omega^{\prime}, \omega^{\prime \prime}\right\}}}\left(\Psi_{e}\right) \wedge \varphi_{\left\{\omega^{\prime}, \omega^{\prime \prime}\right\}} \equiv \diamond_{\varphi_{\left\{\omega^{\prime}, \omega^{\prime \prime}\right\}}}\left(\Psi_{e}\right)$ but $\omega^{\prime} \not \models \diamond_{\varphi_{\left\{\omega, \omega^{\prime}, \omega^{\prime \prime}\right\}}}\left(\Psi_{e}\right)$ so $\omega^{\prime} \not \models \diamond_{\varphi_{\left\{\omega^{\prime}, \omega^{\prime \prime}\right\}}}\left(\Psi_{e}\right)$. Contradiction.

Case $2 \diamond_{\varphi_{\left\{\omega, \omega^{\prime}, \omega^{\prime \prime}\right\}}}\left(\Psi_{e}\right) \wedge \varphi_{\left\{\omega, \omega^{\prime \prime}\right\}}$ is not consistent. So $\diamond_{\varphi_{\left\{\omega, \omega^{\prime}, \omega^{\prime \prime}\right\}}}\left(\Psi_{e}\right)=\varphi_{\left\{\omega^{\prime}\right\}}$. Then $\diamond_{\varphi_{\left\{\omega, \omega^{\prime}, \omega^{\prime \prime}\right\}}}\left(\Psi_{e}\right) \wedge \varphi_{\left\{\omega, \omega^{\prime}\right\}}=\varphi_{\left\{\omega^{\prime}\right\}}$. By (UC7) and (UC8) it follows that $\diamond_{\varphi_{\left\{\omega, \omega^{\prime}\right\}}}\left(\Psi_{e}\right)=\varphi_{\left\{\omega^{\prime}\right\}}$, that means by definition $\omega^{\prime}<\Psi_{e} \omega$. Contradiction.

Now we show that $\bmod \left(\diamond_{\mu}(\Psi)\right)=\bigcup_{e \models \Psi} \min \left(\bmod (\mu), \leq_{e}\right)$. In case $\Psi$ is not pconsistent, previous equation follows in a straightforward way of postulate (UC1) part (b). Now we consider the case in which $\Psi$ is p-consistent. First for the inclusion $\left.\bmod \left(\diamond_{\mu}(\Psi)\right) \subseteq \bigcup_{e \models \Psi} \min (\bmod (\omega)), \leq_{e}\right)$ assume that $\omega \models \diamond_{\mu}(\Psi)$ and suppose towards a contradiction that $\omega \notin \bigcup_{e \models \Psi} \min \left(\bmod (\mu), \leq_{e}\right)$. So we can find for each $e$ a $\omega^{\prime} \models \mu$ s.t. $\omega^{\prime}<_{e} \omega$, then $\omega \not \models \diamond_{\varphi_{\left\{\omega, \omega^{\prime}\right\}}}\left(\Psi_{e}\right)$. Since $\diamond_{\mu}(\Psi) \wedge \varphi_{\left\{\omega, \omega^{\prime}\right\}}$ is consistent, from (UC7) and (UC8) we have $\diamond_{\mu}(\Psi) \wedge \varphi_{\left\{\omega, \omega^{\prime}\right\}} \equiv \diamond_{\varphi_{\left\{\omega, \omega^{\prime}\right\}}}(\Psi)$. But $\omega \not \models \diamond_{\varphi_{\left\{\omega, \omega^{\prime}\right\}}}\left(\Psi_{e}\right)$ for all $e$, so $\omega \not \models \diamond_{\varphi_{\left\{\omega, \omega^{\prime}\right\}}}(\Psi)$. Thus $\omega \not \models \diamond_{\mu}(\Psi)$. Contradiction.

For the other inclusion $\bmod \left(\diamond_{\mu}(\Psi)\right) \supseteq \bigcup_{e \models \Psi} \min \left(\bmod (\mu), \leq_{e}\right)$, suppose that $\exists e \models$ $\Psi$ such that $\omega \in \min \left(\bmod (\mu), \leq_{e}\right)$. We want to show that $\omega \models \diamond_{\mu}(\Psi)$. Since $\omega \in$ $\min \left(\bmod (\mu), \leq_{e}\right), \forall \omega^{\prime} \models \mu, \omega \leq_{e} \omega^{\prime}$ and so $\omega \models \diamond_{\varphi_{\left\{\omega, \omega^{\prime}\right\}}}\left(\Psi_{e}\right)$. Since $\diamond_{\mu}\left(\Psi_{e}\right) \wedge \varphi_{\left\{\omega, \omega^{\prime}\right\}}$ is consistent from (UC7) and (UC8) we have $\diamond_{\mu}\left(\Psi_{e}\right) \wedge \varphi_{\left\{\omega, \omega^{\prime}\right\}} \equiv \diamond_{\varphi_{\left\{\omega, \omega^{\prime}\right\}}}\left(\Psi_{e}\right)$. Since $\omega \models \diamond_{\varphi_{\left\{\omega, \omega^{\prime}\right\}}}\left(\Psi_{e}\right)$, by the previous equivalence, $\omega \models \diamond_{\mu}\left(\Psi_{e}\right)$, and, by Lemma 1 (note that here we find the utilization of (UC9)) this implies that $\omega \models \diamond_{\mu}(\Psi)$.

It remains to verify the conditions of the distributed assignment:

1 Consider $\omega$ and define $e=\{\omega, \ldots, \omega\}$. So as $\bigwedge \Psi_{e} \equiv \varphi_{\{\omega\}}, \bigwedge \Psi_{e} \vdash \varphi_{\left\{\omega, \omega^{\prime}\right\}}$, then by $(\mathrm{UC} 2) \wedge \Psi_{e} \equiv \diamond_{\varphi_{\left\{\omega, \omega^{\prime}\right\}}}\left(\Psi_{e}\right)$. So $\bmod \left(\diamond_{\varphi_{\left\{\omega, \omega^{\prime}\right\}}}\left(\Psi_{e}\right)\right)=\{\omega\}$. So $\omega<_{e} \omega^{\prime}$.

2 Since that $\leq_{\left\{\omega, \omega^{\prime}\right\}}$ is a total preorder, in order to prove $\omega \simeq_{\left\{\omega, \omega^{\prime}\right\}} \omega^{\prime}$, it is enough to see that $\omega \leq_{\left\{\omega, \omega^{\prime}\right\}} \omega^{\prime} \Leftrightarrow \omega^{\prime} \leq_{\left\{\omega, \omega^{\prime}\right\}} \omega$. Take $\mu=\varphi_{\left\{\omega, \omega^{\prime}\right\}}$ and $e=\left\{\omega, \omega^{\prime}\right\}$. Then $\varphi_{\{\omega\}} \vdash$ $\mu$ and $\varphi_{\left\{\omega^{\prime}\right\}} \vdash \mu$. But observe that $\omega \leq_{\left\{\omega, \omega^{\prime}\right\}} \omega^{\prime} \Longleftrightarrow \omega \models \diamond_{\mu}\left(\Psi_{e}\right) \Longleftrightarrow \diamond_{\mu}\left(\Psi_{e}\right) \wedge$

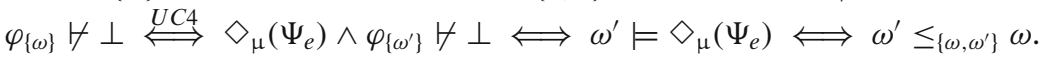

3 If $\omega \leq_{e_{1}} \omega^{\prime}$ and $\omega \leq_{e_{2}} \omega^{\prime}$, this means by definition that $\omega \models \diamond_{\varphi_{\left\{\omega, \omega^{\prime}\right\}}}\left(\Psi_{e_{1}}\right)$ and $\omega \models$ $\diamond_{\varphi_{\left\{\omega, \omega^{\prime}\right\}}}\left(\Psi_{e_{2}}\right)$. So by (UC5) we know that $\omega \models \diamond_{\varphi_{\left\{\omega, \omega^{\prime}\right\}}}\left(\Psi_{e_{1}} \sqcup \Psi_{e_{2}}\right)$, that by definition means $\omega \leq_{e_{1} \sqcup e_{2}} \omega^{\prime}$. 
4 If $\omega<_{e_{1}} \omega^{\prime}$ and $\omega \leq_{e_{2}} \omega^{\prime}$ this means that by definition $\omega \models \diamond_{\varphi_{\left\{\omega, \omega^{\prime}\right\}}}\left(\Psi_{e_{1}}\right)$, that $\omega^{\prime} \not \models \diamond_{\varphi_{\left\{\omega, \omega^{\prime}\right\}}}\left(\Psi_{e_{1}}\right)$ and $\omega \models \diamond_{\varphi_{\left\{\omega, \omega^{\prime}\right\}}}\left(\Psi_{e_{2}}\right)$. So $\diamond_{\varphi_{\left\{\omega, \omega^{\prime}\right\}}}\left(\Psi_{e_{1}}\right) \wedge \diamond_{\varphi_{\left\{\omega, \omega^{\prime}\right\}}}\left(\Psi_{e_{2}}\right)$ is consistent (with $\omega$ as unique model), and by (UC5) and (UC6) we have in this case $\bmod \left(\diamond_{\varphi_{\left\{\omega, \omega^{\prime}\right\}}}\left(\Psi_{e_{1}} \sqcup \Psi_{e_{2}}\right)\right)=\omega$. By definition this means that $\omega<_{e_{1} \sqcup e_{2}} \omega^{\prime}$.

(If part) Let's consider a distributed assignment that maps each state $e$ to a total preorder $\leq_{e}$ and define an operator $\diamond$ by putting $\bmod \left(\diamond_{\mu}(\Psi)\right)=\bigcup_{e \models \Psi}\left(\min \left(\bmod (\mu), \leq_{e}\right.\right.$ ). We want to show that $\diamond$ satisfies (UC0-UC9).

(UC0) By definition $\bmod \left(\diamond_{\mu}(\Psi)\right) \subseteq \bmod (\mu)$.

(UC1) (a) If $\mu$ is consistent, and $\Psi$ is p-consistent, then $\bmod (\mu) \neq \emptyset$ and there exists a state $e$ of $\Psi$. Moreover, for each $e$ of $\Psi$, as we have a finite number of interpretations, we have no infinite descending chains with respect to $<_{e}$, so $\min \left(\bmod (\mu), \leq_{e}\right) \neq \emptyset$. Then $\diamond_{\mu}(\Psi)$ is consistent.

(b) If $\Psi$ is not p-consistent, then, for any $\mu, \bigcup_{e \models \Psi} \min \left(\bmod (\mu), \leq_{e}\right)=\emptyset$. Thus $\bmod \left(\diamond_{\mu}(\Psi)\right)=\emptyset$. Therefore $\diamond_{\mu}(\Psi)$ is inconsistent.

(UC2) Assume that $\bigwedge \Psi$ is consistent, and that $\bigwedge \Psi \vdash \mu$. So for any $\omega \models$ $\bigwedge \Psi$, we have $e=\{\omega, \ldots, \omega\} \models \Psi$. By condition 1 we deduce that $\omega \in$ $\min \left(\bmod (\mu), \leq_{e}\right)$. So $\bigwedge \Psi \vdash \diamond_{\mu}(\Psi)$.

(UC3) Direct from the definition of $\diamond$.

(UC5) If $\omega \models \diamond_{\mu}\left(\Psi_{1}\right) \wedge \diamond_{\mu}\left(\Psi_{2}\right)$ then $\exists e_{1} \models \Psi_{1}$ such that $\omega \in \min \left(\bmod (\mu), \leq_{e_{1}}\right)$ and so $\forall \omega^{\prime} \models \mu \omega \leq_{e_{1}} \omega^{\prime}$. We have in the same way $\exists e_{2} \models \Psi_{2}$ such that $\forall \omega^{\prime} \models \mu \omega \leq_{e_{2}} \omega^{\prime}$. So by condition 2 we have that $\forall \omega^{\prime} \models \mu \omega \leq_{e_{1} \sqcup e_{2}} \omega^{\prime}$. So $\omega \in \min \left(\bmod (\mu), \leq_{e_{1} \sqcup e_{2}}\right)$. So, as $e_{1} \sqcup e_{2} \models \Psi_{1} \sqcup \Psi_{2}$, by definition $\omega \models$ $\diamond_{\mu}\left(\Psi_{1} \sqcup \Psi_{2}\right)$.

(UC6) Assume that $\Psi_{1}$ and $\Psi_{2}$ are complete profile and that $\diamond_{\mu}\left(\Psi_{1}\right) \wedge \diamond_{\mu}\left(\Psi_{2}\right)$ is consistent. We want to show that $\diamond_{\mu}\left(\Psi_{1} \sqcup \Psi_{2}\right) \vdash \diamond_{\mu}\left(\Psi_{1}\right) \wedge \diamond_{\mu}\left(\Psi_{2}\right)$ holds. Take $\omega \models \diamond_{\mu}\left(\Psi_{1} \sqcup \Psi_{2}\right)$. As $\Psi_{1}$ and $\Psi_{2}$ are complete profile there is a unique state for each profile, let us denote them respectively $e_{1}^{*}$ and $e_{2}^{*}$. So $\forall \omega^{\prime} \models \mu \omega \leq e_{1}^{*} \sqcup e_{2}^{*} \omega^{\prime}$. Suppose towards a contradiction that $\omega \not \models \diamond_{\mu}\left(\Psi_{1}\right) \wedge$ $\diamond_{\mu}\left(\Psi_{2}\right)$. So $\omega \not \models \diamond_{\mu}\left(\Psi_{1}\right)$ or $\omega \not \models \diamond_{\mu}\left(\Psi_{2}\right)$. Suppose that $\omega \not \models \diamond_{\mu}\left(\Psi_{1}\right)$ (the other case is symmetrical). This means that, for all $\omega^{\prime} \in \min \left(\bmod (\mu), \leq_{e_{1}^{*}}\right)$, $\omega^{\prime}<_{e_{1}^{*}} \omega$. As $\diamond_{\mu}\left(\Psi_{1}\right) \wedge \diamond_{\mu}\left(\Psi_{2}\right)$ is consistent $\exists \omega^{\prime} \models \diamond_{\mu}\left(\Psi_{1}\right) \wedge \diamond_{\mu}\left(\Psi_{2}\right)$. So $\omega^{\prime} \in \min \left(\bmod (\mu), \leq_{e_{1}^{*}}\right)$ and $\left.\omega^{\prime} \in \min (\bmod (\omega)), \leq_{e_{2}^{*}}\right)$. So we have that $\omega^{\prime}<_{e_{1}^{*}}$ $\omega$ and $\omega^{\prime} \leq_{e_{2}^{*}} \omega$. And by condition 3 we deduce $\omega^{\prime}<_{e_{1}^{*} \sqcup e_{2}^{*}} \omega$. So, as $e_{1}^{*} \sqcup e_{2}^{*}$ is the only state of $\Psi_{1} \sqcup \Psi_{2}$, this means that $\omega \not \models \diamond_{\mu}\left(\Psi_{1} \sqcup \Psi_{2}\right)$. Contradiction.

(UC7) Let's take $\omega \models \diamond_{\mu_{1}}(\Psi) \wedge \mu_{2}$. So this means that $\exists e \models \Psi$ s.t. $\forall \omega^{\prime} \models \mu_{1} \omega \leq_{e}$ $\omega^{\prime}$. So $\forall \omega^{\prime} \models \mu_{1} \wedge \mu_{2} \omega \leq_{e} \omega^{\prime}$, so $\omega \models \diamond_{\mu_{1} \wedge \mu_{2}}(\Psi)$.

(UC8) Assume that $\diamond_{\mu_{1}}(\Psi) \wedge \mu_{2}$ is consistent, so $\exists \omega^{\prime} \models \diamond_{\mu_{1}}(\Psi) \wedge \mu_{2}$. Consider $\omega \models \diamond_{\mu_{1} \wedge \mu_{2}}(\Psi)$ and suppose that $\omega \not \models \diamond_{\mu_{1}}(\Psi) \wedge \mu_{2}$. As $\omega \models \mu_{2}$ then $\omega \not \models$ $\diamond_{\mu_{1}}(\Psi)$. As $\Psi$ is a complete profile then there is a unique state $e$ for this profile, and $\omega^{\prime}<_{e} \omega$. As $\omega^{\prime} \models \mu_{1} \wedge \mu_{2}$ then $\omega \notin \min \left(\bmod \left(\mu_{1} \wedge \mu_{2}\right), \leq_{e}\right)$. Thus $\omega \not \models \diamond_{\mu_{1} \wedge \mu_{2}}(\Psi)$. Contradiction.

(UC4) Suppose that $\varphi$ and $\varphi^{\prime}$ are complete formulas such that $\varphi \vdash \mu, \varphi^{\prime} \vdash \mu$, and $\diamond_{\mu}\left(\varphi \sqcup \varphi^{\prime}\right) \wedge \varphi \nvdash \perp$, we want to show that $\diamond_{\mu}\left(\varphi \sqcup \varphi^{\prime}\right) \wedge \varphi^{\prime} \nvdash \perp$. Since $\varphi$ and $\varphi^{\prime}$ are complete formulas by UC3, we can suppose that $\varphi=\varphi_{\{\omega\}}$ and $\varphi^{\prime}=\varphi_{\left\{\omega^{\prime}\right\}}$. Let $e=\left(\omega, \omega^{\prime}\right)$. From the assumption $\diamond_{\mu}\left(\Psi_{e}\right) \wedge \varphi_{\{\omega\}} \nvdash \perp$, it is easy to see by UC7, UC8 and UC3 that $\diamond_{\mu}\left(\Psi_{e}\right) \wedge \varphi_{\left\{\omega, \omega^{\prime}\right\}} \equiv \diamond_{\varphi_{\left\{\omega, \omega^{\prime}\right\}}}\left(\Psi_{e}\right)(*)$ and since $\omega \models \diamond_{\mu}\left(\Psi_{e}\right), \omega \models \diamond_{\varphi_{\left\{\omega, \omega^{\prime}\right\}}}\left(\Psi_{e}\right)$. By the condition 2, the fact that 
$\omega \models \diamond_{\varphi_{\left\{\omega, \omega^{\prime}\right\}}}\left(\Psi_{e}\right)$ and the definition of $\diamond_{\varphi_{\left\{\omega, \omega^{\prime}\right\}}}\left(\Psi_{e}\right), \omega^{\prime} \models \diamond_{\varphi_{\left\{\omega, \omega^{\prime}\right\}}}\left(\Psi_{e}\right)$. From this and $(*)$, it follows $\omega^{\prime} \models \diamond_{\mu}\left(\Psi_{e}\right)$ that is $\diamond_{\mu}\left(\Psi_{e}\right) \wedge \varphi_{\left\{\omega^{\prime}\right\}} \nvdash \perp$.

(UC9) Note that by definition $e \models \Psi \sqcup\left\{\varphi \vee \varphi^{\prime}\right\}$ iff $\left(e \models \Psi \sqcup\{\varphi\}\right.$ or $\left.e \models \Psi \sqcup\left\{\varphi^{\prime}\right\}\right)$. So if $\omega \models \diamond_{\mu}\left(\Psi \sqcup\left\{\varphi \vee \varphi^{\prime}\right\}\right)$, this means that there is a state $e \models \Psi \sqcup\left\{\varphi \vee \varphi^{\prime}\right\}$ such that $\forall \omega^{\prime} \omega \leq_{e} \omega^{\prime}$. As $e \models \Psi \sqcup\{\varphi\}$ or $e \models \Psi \sqcup\left\{\varphi^{\prime}\right\}$, we have by definition $\omega \models \diamond_{\mu}(\Psi \sqcup\{\varphi\})$ or $\omega \models \diamond_{\mu}\left(\Psi \sqcup\left\{\varphi^{\prime}\right\}\right)$. Similarly if $\omega \models \diamond_{\mu}(\Psi \sqcup\{\varphi\})$ or $\omega \models$ $\diamond_{\mu}\left(\Psi \sqcup\left\{\varphi^{\prime}\right\}\right)$, then $\omega \models \Psi \sqcup\left\{\varphi \vee \varphi^{\prime}\right\}$.

Proof of Theorem 8 Most of the update postulates are obtained directly from confluence ones while considering singleton profiles. (U1) is direct from (UC0). (U3) is direct from (UC1) part (a). (U4) is direct from (UC3). (U5) is direct from (UC7). (U8) is direct from (UC9). (U9) is direct from (UC8).

(U2) Suppose that $\varphi \vdash \mu$. If $\varphi \vdash \perp$ then by (UC1) part (b), $\diamond_{\mu}(\varphi) \equiv \perp$, that is $\diamond_{\mu}(\varphi) \equiv \varphi$.

If $\varphi \nvdash \perp$, then let us note $\omega_{1}, \ldots, \omega_{n}$ the models of $\varphi$. Then $\varphi=\varphi_{\left\{\omega_{1}\right\}} \vee \ldots \vee \varphi_{\left\{\omega_{n}\right\}}$. Then by (UC9) we have that $\diamond_{\mu}(\{\varphi\})=\diamond_{\mu}\left(\varphi_{\left\{\omega_{1}\right\}}\right) \vee \ldots \diamond_{\mu}\left(\varphi_{\left\{\omega_{n}\right\}}\right)$. Now by (UC2) we have that for each $i, \diamond_{\mu}\left(\varphi_{\left\{\omega_{1}\right\}}\right)=\varphi_{\left\{\omega_{1}\right\}}$. So this gives $\diamond_{\mu}(\{\varphi\})=\varphi_{\left\{\omega_{1}\right\}} \vee \ldots \vee \varphi_{\left\{\omega_{n}\right\}}$, i.e., $\diamond_{\mu}(\{\varphi\})=\varphi$, that by definition is $\varphi \diamond \mu \equiv \varphi$.

(U6) and (U7) are consequence of previous postulates.

Proof of Theorem 9 The first part is an easy consequence of Representations Theorems 4 and 7 . It is enough to note that a syncretic assignment specialized to complete profiles becomes a distributed assignment.

The second part is not as straightforward as Theorem 5's proof. We need something more than the established Representations Theorems. In fact, given a distributed assignment representing the confluence operator, we need to build a syncretic assignment extending this given distributed assignment. In order to do that we can use the following construction: Each pre-order $\leq_{e}$ defines naturally a (integer) rank function $r_{e}$ on interpretations in the following way: $r_{e}(\omega)=n$ if $n$ is the length of the longest sequence $\left(\omega_{0}, \ldots, \omega_{n}\right)$ such that $\omega_{0}<_{e} \ldots<_{e} \omega_{n-1}<_{e} \omega_{n}$, with $\omega=\omega_{n}$.

Then we put $\omega \leq_{\Psi} \omega^{\prime}$ if and only if $\quad \sum_{e \models \Psi} r_{e}(\omega) \leq \sum_{e \models \Psi} r_{e}\left(\omega^{\prime}\right)$.

We can verify easily that this is a syncretic assignment extending the distributed assignment. Therefore, if $\diamond$ is the confluence operator given by the distributed assignment and $\Delta$ is the merging operator given by the syncretic assignment defined above, they satisfy (2).

\section{Proof of Theorem 10}

If Suppose that a distance-based operator $\diamond^{d, f}$ is defined from an aggregation function $f$ satisfying composition and decomposition. Let us check that this operator satisfies (UC0-UC9). We will show that the pre-order $\leq_{e}$ defined from this operator (see Definition 12) is a distributed assignment, then we will use the representation theorem (Theorem 7) to conclude.

1. $\omega<_{\{\omega, \ldots, \omega\}} \omega^{\prime}$ if $\omega^{\prime} \neq \omega$.

By definition of the distance we have that $d\left(\omega, \omega^{\prime}\right)=0$ if and only if $\omega=\omega^{\prime}$, and from the minimality property of the aggregation function we have that $f\left(x_{1}, \ldots\right.$, 
$\left.x_{n}\right)=0$ if and only if $x_{1}=\ldots=x_{n}=0$. So $f(d(\omega, \omega), \ldots, d(\omega, \omega))=0$ and for any $\omega^{\prime} \neq \omega$ then $f\left(d\left(\omega, \omega^{\prime}\right), \ldots, d\left(\omega, \omega^{\prime}\right)\right)>0$. So $\omega<_{\{\omega, \ldots, \omega\}} \omega^{\prime}$.

2. $\omega \simeq\left\{\omega, \omega^{\prime}\right\} \omega^{\prime}$.

$d\left(\omega,\left\{\omega, \omega^{\prime}\right\}\right)=f\left(d(\omega, \omega), d\left(\omega, \omega^{\prime}\right)\right)=f\left(0, d\left(\omega, \omega^{\prime}\right)\right)$.

$d\left(\omega^{\prime},\left\{\omega, \omega^{\prime}\right\}\right)=f\left(d\left(\omega^{\prime}, \omega\right), d\left(\omega^{\prime}, \omega^{\prime}\right)\right)=f\left(d\left(\omega^{\prime}, \omega\right), 0\right)$.

From symmetry of the aggregation function we have that $f\left(0, d\left(\omega, \omega^{\prime}\right)\right)=$ $f\left(d\left(\omega, \omega^{\prime}\right), 0\right)$. By the property of the distance we have that $d\left(\omega, \omega^{\prime}\right)=d\left(\omega^{\prime}, \omega\right)$. So $f\left(0, d\left(\omega, \omega^{\prime}\right)\right)=f\left(d\left(\omega^{\prime}, \omega\right), 0\right)$, so $d\left(\omega,\left\{\omega, \omega^{\prime}\right\}\right)=d\left(\omega^{\prime},\left\{\omega, \omega^{\prime}\right\}\right)$. That is the definition of $\omega \simeq\left\{\omega, \omega^{\prime}\right\} \omega^{\prime}$.

3. If $\omega \leq_{e_{1}} \omega^{\prime}$ and $\omega \leq_{e_{2}} \omega^{\prime}$, then $\omega \leq_{e_{1} \sqcup e_{2}} \omega^{\prime}$

Let us note $e_{1}=\left\{\omega_{1}^{1}, \ldots, \omega_{n_{1}}^{1}\right\}$. From $\omega \leq_{e_{1}} \omega^{\prime}$ we have that

$$
f\left(d\left(\omega, \omega_{1}^{1}\right), \ldots, d\left(\omega, \omega_{n_{1}}^{1}\right)\right) \leq f\left(d\left(\omega^{\prime}, \omega_{1}^{1}\right), \ldots, d\left(\omega^{\prime}, \omega_{n_{1}}^{1}\right)\right)
$$

Similarly, if we note $e_{2}=\left\{\omega_{1}^{2}, \ldots, \omega_{n_{1}}^{2}\right\}$, we have from $\omega \leq_{e_{2}} \omega^{\prime}$ :

$$
f\left(d\left(\omega, \omega_{1}^{2}\right), \ldots, d\left(\omega, \omega_{n_{1}}^{2}\right)\right) \leq f\left(d\left(\omega^{\prime}, \omega_{1}^{2}\right), \ldots, d\left(\omega^{\prime}, \omega_{n_{1}}^{2}\right)\right)
$$

Now by applying composition several times to the inequality (3), we obtain:

$$
\begin{aligned}
& f\left(d\left(\omega, \omega_{1}^{1}\right), \ldots, d\left(\omega, \omega_{n_{1}}^{1}\right), d\left(\omega, \omega_{1}^{2}\right), \ldots, d\left(\omega, \omega_{n_{1}}^{2}\right)\right) \\
& \quad \leq f\left(d\left(\omega^{\prime}, \omega_{1}^{1}\right), \ldots, d\left(\omega^{\prime}, \omega_{n_{1}}^{1}\right), d\left(\omega, \omega_{1}^{2}\right), \ldots, d\left(\omega, \omega_{n_{1}}^{2}\right)\right)
\end{aligned}
$$

Similarly, if we apply composition several times to the inequality (4), we obtain:

$$
\begin{aligned}
& f\left(d\left(\omega, \omega_{1}^{2}\right), \ldots, d\left(\omega, \omega_{n_{1}}^{2}\right), d\left(\omega^{\prime}, \omega_{1}^{1}\right), \ldots, d\left(\omega^{\prime}, \omega_{n_{1}}^{1}\right)\right) \\
& \quad \leq f\left(d\left(\omega^{\prime}, \omega_{1}^{2}\right), \ldots, d\left(\omega^{\prime}, \omega_{n_{1}}^{2}\right), d\left(\omega^{\prime}, \omega_{1}^{1}\right), \ldots, d\left(\omega^{\prime}, \omega_{n_{1}}^{1}\right)\right)
\end{aligned}
$$

Now, by symmetry, we have that

$$
\begin{aligned}
f & \left(d\left(\omega^{\prime}, \omega_{1}^{1}\right), \ldots, d\left(\omega^{\prime}, \omega_{n_{1}}^{1}\right), d\left(\omega, \omega_{1}^{2}\right), \ldots, d\left(\omega, \omega_{n_{1}}^{2}\right)\right) \\
& =f\left(d\left(\omega, \omega_{1}^{2}\right), \ldots, d\left(\omega, \omega_{n_{1}}^{2}\right), d\left(\omega^{\prime}, \omega_{1}^{1}\right), \ldots, d\left(\omega^{\prime}, \omega_{n_{1}}^{1}\right)\right) .
\end{aligned}
$$

So, now using transitivity, we obtain that

$$
\begin{aligned}
& f\left(d\left(\omega, \omega_{1}^{1}\right), \ldots, d\left(\omega, \omega_{n_{1}}^{1}\right), d\left(\omega, \omega_{1}^{2}\right), \ldots, d\left(\omega, \omega_{n_{1}}^{2}\right)\right) \\
& \quad \leq f\left(d\left(\omega^{\prime}, \omega_{1}^{2}\right), \ldots, d\left(\omega^{\prime}, \omega_{n_{1}}^{2}\right), d\left(\omega^{\prime}, \omega_{1}^{1}\right), \ldots, d\left(\omega^{\prime}, \omega_{n_{1}}^{1}\right)\right)
\end{aligned}
$$

Using symmetry again, we finally obtain:

$$
\begin{aligned}
& f\left(d\left(\omega, \omega_{1}^{1}\right), \ldots, d\left(\omega, \omega_{n_{1}}^{1}\right), d\left(\omega, \omega_{1}^{2}\right), \ldots, d\left(\omega, \omega_{n_{1}}^{2}\right)\right) \\
& \quad \leq f\left(d\left(\omega^{\prime}, \omega_{1}^{1}\right), \ldots, d\left(\omega^{\prime}, \omega_{n_{1}}^{1}\right), d\left(\omega^{\prime}, \omega_{1}^{2}\right), \ldots, d\left(\omega^{\prime}, \omega_{n_{1}}^{2}\right)\right)
\end{aligned}
$$

That is exactly $\omega \leq_{e_{1} \sqcup e_{2}} \omega^{\prime}$.

4. If $\omega<_{e_{1}} \omega^{\prime}$ and $\omega \leq_{e_{2}} \omega^{\prime}$, then $\omega<_{e_{1} \sqcup e_{2}} \omega^{\prime}$

This proof is similar as the one of the previous condition. First, we have just note that decomposition is equivalent to the following condition:

If $f\left(x_{1}, \ldots, x_{n}\right)<f\left(y_{1}, \ldots, y_{n}\right)$, then $f\left(x_{1}, \ldots, x_{n}, z\right)<f\left(y_{1}, \ldots, y_{n}, z\right)$. 
Let us note $e_{1}=\left\{\omega_{1}^{1}, \ldots, \omega_{n_{1}}^{1}\right\}$. Now from $\omega<_{e_{1}} \omega^{\prime}$ we have that

$$
f\left(d\left(\omega, \omega_{1}^{1}\right), \ldots, d\left(\omega, \omega_{n_{1}}^{1}\right)\right)<f\left(d\left(\omega^{\prime}, \omega_{1}^{1}\right), \ldots, d\left(\omega^{\prime}, \omega_{n_{1}}^{1}\right)\right)
$$

Similarly if we note $e_{2}=\left\{\omega_{1}^{2}, \ldots, \omega_{n_{1}}^{2}\right\}$, we have from $\omega \leq_{e_{2}} \omega^{\prime}$ :

$$
f\left(d\left(\omega, \omega_{1}^{2}\right), \ldots, d\left(\omega, \omega_{n_{1}}^{2}\right)\right) \leq f\left(d\left(\omega^{\prime}, \omega_{1}^{2}\right), \ldots, d\left(\omega^{\prime}, \omega_{n_{1}}^{2}\right)\right)
$$

Now, by applying decomposition several times to the inequality (5), we obtain:

$$
\begin{aligned}
& f\left(d\left(\omega, \omega_{1}^{1}\right), \ldots, d\left(\omega, \omega_{n_{1}}^{1}\right), d\left(\omega, \omega_{1}^{2}\right), \ldots, d\left(\omega, \omega_{n_{1}}^{2}\right)\right) \\
& \quad<f\left(d\left(\omega^{\prime}, \omega_{1}^{1}\right), \ldots, d\left(\omega^{\prime}, \omega_{n_{1}}^{1}\right), d\left(\omega, \omega_{1}^{2}\right), \ldots, d\left(\omega, \omega_{n_{1}}^{2}\right)\right)
\end{aligned}
$$

Similarly, if we apply composition several times to the inequality (6), we obtain:

$$
\begin{aligned}
& f\left(d\left(\omega, \omega_{1}^{2}\right), \ldots, d\left(\omega, \omega_{n_{1}}^{2}\right), d\left(\omega^{\prime}, \omega_{1}^{1}\right), \ldots, d\left(\omega^{\prime}, \omega_{n_{1}}^{1}\right)\right) \\
& \quad \leq f\left(d\left(\omega^{\prime}, \omega_{1}^{2}\right), \ldots, d\left(\omega^{\prime}, \omega_{n_{1}}^{2}\right), d\left(\omega^{\prime}, \omega_{1}^{1}\right), \ldots, d\left(\omega^{\prime}, \omega_{n_{1}}^{1}\right)\right)
\end{aligned}
$$

Now, by symmetry we have

$$
\begin{aligned}
f & \left(d\left(\omega^{\prime}, \omega_{1}^{1}\right), \ldots, d\left(\omega^{\prime}, \omega_{n_{1}}^{1}\right), d\left(\omega, \omega_{1}^{2}\right), \ldots, d\left(\omega, \omega_{n_{1}}^{2}\right)\right) \\
& =f\left(d\left(\omega, \omega_{1}^{2}\right), \ldots, d\left(\omega, \omega_{n_{1}}^{2}\right), d\left(\omega^{\prime}, \omega_{1}^{1}\right), \ldots, d\left(\omega^{\prime}, \omega_{n_{1}}^{1}\right)\right)
\end{aligned}
$$

and by transitivity, we obtain

$$
\begin{aligned}
& f\left(d\left(\omega, \omega_{1}^{1}\right), \ldots, d\left(\omega, \omega_{n_{1}}^{1}\right), d\left(\omega, \omega_{1}^{2}\right), \ldots, d\left(\omega, \omega_{n_{1}}^{2}\right)\right) \\
& \quad<f\left(d\left(\omega^{\prime}, \omega_{1}^{2}\right), \ldots, d\left(\omega^{\prime}, \omega_{n_{1}}^{2}\right), d\left(\omega^{\prime}, \omega_{1}^{1}\right), \ldots, d\left(\omega^{\prime}, \omega_{n_{1}}^{1}\right)\right)
\end{aligned}
$$

Using symmetry again, we finally obtain:

$$
\begin{aligned}
& f\left(d\left(\omega, \omega_{1}^{1}\right), \ldots, d\left(\omega, \omega_{n_{1}}^{1}\right), d\left(\omega, \omega_{1}^{2}\right), \ldots, d\left(\omega, \omega_{n_{1}}^{2}\right)\right) \\
& \quad<f\left(d\left(\omega^{\prime}, \omega_{1}^{1}\right), \ldots, d\left(\omega^{\prime}, \omega_{n_{1}}^{1}\right), d\left(\omega^{\prime}, \omega_{1}^{2}\right), \ldots, d\left(\omega^{\prime}, \omega_{n_{1}}^{2}\right)\right)
\end{aligned}
$$

That is exactly $\omega<_{e_{1} \sqcup e_{2}} \omega^{\prime}$.

Proof of Theorem 11 The proof of the if part is similar to Theorem 10's proof.

Only if Suppose that we have a distance-based confluence operator $\diamond^{d, f}$ that satisfies (UC0-UC9). Let us show that the aggregation function satisfies $d$-composition and $d$-decomposition.

$d$-composition. Let $(\vec{x}, \vec{y}, z)$ be a $d$-realizable triple. Suppose $f\left(x_{1}, \ldots, x_{n}\right) \leq f\left(y_{1}\right.$, $\left.\ldots, y_{n}\right)$. Consider two interpretations $\omega, \omega^{\prime}$ such that for every $i \in 1, \ldots, n$, $d\left(\omega, \omega_{i}\right)=x_{i}$ and $d\left(\omega^{\prime}, \omega_{i}\right)=y_{i}$. From the definition of the distance-based confluence operators, we have $\omega \models \diamond_{\text {form }\left(\left\{\omega, \omega^{\prime}\right\}\right)}\left(\left\{\varphi_{\left\{\omega_{1}\right\}}, \ldots, \varphi_{\left\{\omega_{n}\right\}}\right\}\right)$. Now let us take an interpretation $\omega^{\prime \prime}$, such that $d\left(\omega, \omega^{\prime \prime}\right)=d\left(\omega^{\prime}, \omega^{\prime \prime}\right)=z$, we have both $\omega \models$ $\diamond_{\text {form }\left(\left\{\omega, \omega^{\prime}\right\}\right)}\left(\left\{\varphi_{\left\{\omega^{\prime \prime}\right\}}\right\}\right)$ and $\omega^{\prime} \models \diamond_{\text {form }\left(\left\{\omega, \omega^{\prime}\right\}\right)}\left(\left\{\varphi_{\left\{\omega^{\prime \prime}\right\}}\right\}\right)$. Now, from (UC5), we conclude that $\left.\omega \models \diamond_{\text {form }\left(\left\{\omega, \omega^{\prime}\right\}\right)}\left(\left\{\varphi_{\left\{\omega_{1}\right\}}, \ldots, \varphi_{\left\{\omega_{n}\right\}}, \varphi_{\left\{\omega^{\prime \prime}\right\}}\right\}\right\}\right)$, or equivalently (from the definition of the operators) $f\left(x_{1}, \ldots, x_{n}, z\right) \leq f\left(y_{1}, \ldots, y_{n}, z\right)$. 
$d$-decomposition. Let $(\vec{x}, \vec{y}, z)$ be a $d$-realizable triple. We will show the equivalent condition:

$$
\text { if } f\left(x_{1}, \ldots, x_{n}\right)<f\left(y_{1}, \ldots, y_{n}\right) \text {, then } f\left(x_{1}, \ldots, x_{n}, z\right)<f\left(y_{1}, \ldots, y_{n}, z\right) \text {. }
$$

Suppose $f\left(x_{1}, \ldots, x_{n}\right)<f\left(y_{1}, \ldots, y_{n}\right)$. Consider two interpretations $\omega, \omega^{\prime}$ such that for every $i \in 1, \ldots, n, d\left(\omega, \omega_{i}\right)=x_{i}$ and $d\left(\omega^{\prime}, \omega_{i}\right)=y_{i}$. From the definition of the distance-based confluence operators, we have

$$
\omega \models \diamond_{\varphi_{\left\{\omega, \omega^{\prime}\right\}}}\left(\left\{\varphi_{\left\{\omega_{1}\right\}}, \ldots, \varphi_{\left\{\omega_{n}\right\}}\right\}\right) \text { and } \omega^{\prime} \not \models \diamond_{\varphi_{\left\{\omega, \omega^{\prime}\right\}}}\left(\left\{\varphi_{\left\{\omega_{1}\right\}}, \ldots, \varphi_{\left\{\omega_{n}\right\}}\right\}\right)
$$

Now let us take an interpretation $\omega^{\prime \prime}$, such that $d\left(\omega, \omega^{\prime \prime}\right)=d\left(\omega^{\prime}, \omega^{\prime \prime}\right)=z$, we have $\omega \models \diamond_{\varphi_{\left\{\omega, \omega^{\prime}\right\}}}\left(\left\{\varphi_{\left\{\omega^{\prime \prime}\right\}}\right\}\right)$ and $\omega^{\prime} \models \diamond_{\varphi_{\left\{\omega, \omega^{\prime}\right\}}}\left(\left\{\varphi_{\left\{\omega^{\prime \prime}\right\}}\right\}\right)$. Since $\left\{\varphi_{\left\{\omega_{1}\right\}}, \ldots, \varphi_{\left\{\omega_{n}\right\}}\right\}$ and $\left\{\varphi_{\left\{\omega^{\prime \prime}\right\}}\right\}$ are complete profiles and since the formula $\diamond_{\varphi_{\left\{\omega, \omega^{\prime}\right\}}}\left(\left\{\varphi_{\left\{\omega_{1}\right\}}, \ldots, \varphi_{\left\{\omega_{n}\right\}}\right\} \wedge \diamond_{\varphi_{\left\{\omega, \omega^{\prime}\right\}}}\left(\left\{\varphi_{\left\{\omega^{\prime \prime}\right\}}\right\}\right)\right.$ is consistent, we conclude, using (UC6),

$$
\diamond_{\varphi_{\left\{\omega, \omega^{\prime}\right\}}}\left(\left\{\varphi_{\left\{\omega_{1}\right\}}, \ldots, \varphi_{\left\{\omega_{n}\right\}}, \varphi_{\left\{\omega^{\prime \prime}\right\}}\right\}\right) \vdash \diamond_{\varphi_{\left\{\omega, \omega^{\prime}\right\}}}\left(\left\{\varphi_{\left\{\omega_{1}\right\}}, \ldots, \varphi_{\left\{\omega_{n}\right\}}\right\}\right) \wedge \diamond_{\varphi_{\left\{\omega, \omega^{\prime}\right\}}}\left(\left\{\varphi_{\left\{\omega^{\prime \prime}\right\}}\right\}\right)
$$

The last formula is equivalent to $\varphi_{\omega}$, so $\diamond_{\varphi_{\left\{\omega, \omega^{\prime}\right\}}}\left(\left\{\varphi_{\left\{\omega_{1}\right\}}, \ldots, \varphi_{\left\{\omega_{n}\right\}}, \varphi_{\left\{\omega^{\prime \prime}\right\}}\right\}\right) \vdash \varphi_{\omega}$ From this and the definition of the operators, we obtain $f\left(x_{1}, \ldots, x_{n}, z\right)<$ $f\left(y_{1}, \ldots, y_{n}, z\right)$ as desired.

Proof of Theorem 13 The first part of the Theorem is an easy verification. It remains to see the that UC9 does not hold. In order to see that, note that a confluence operator $\diamond$ and its majoritarian confluence operator $\diamond$ coincide on complete profiles. It is easy then to check on Example 9, that $\bmod \left(\oplus_{\mu}^{d, \Sigma}\left(\Psi_{e_{6}}\right)\right)=\{0010,0100\}$, and these two interpretations are not included in $\bmod \left(\oplus_{\mu}^{d, \Sigma}(\Psi)\right)=\{1111\}$. That should be the case by Lemma 1 if UC9 was satisfied by $\oplus$.

\section{References}

1. Alchourrón, C., Makinson, D.: On the logic of theory change: Safe contraction. Stud. Log. 44, 405-422 (1985)

2. Alchourrón, C.E., Gärdenfors, P., Makinson D.: On the logic of theory change: Partial meet contraction and revision functions. J. Symb. Log. 50, 510-530 (1985)

3. Baral, C., Kraus, S., Minker, J.: Combining multiple knowledge bases. IEEE Trans. Knowl. Data Eng. 3(2), 208-220 (1991)

4. Baral, C., Kraus, S., Minker, J., Subrahmanian, V.S.: Combining knowledge bases consisting of first-order theories. Comput. Intell. 8(1), 45-71 (1992)

5. Benferhat, S., Lagrue, S., Papini, O.: Revision of partially ordered information: axiomatization, semantics and iteration. In: Proceedings of the Nineteenth International Joint Conference on Artificial Intelligence (IJCAI'05), pp. 376-381 (2005)

6. Booth, R.: Social contraction and belief negotiation. In: Proceedings of the Eighth Conference on Principles of Knowledge Representation and Reasoning (KR'02), pp. 374-384 (2002)

7. Dalal, M.: Investigations into a theory of knowledge base revision: preliminary report. In: Proceedings of the American National Conference on Artificial Intelligence (AAAI'88), pp. 475-479 (1988)

8. Delgrande, J.P., Dubois, D., Lang, J.: Iterated revision as prioritized merging. In: Proceedings of the Tenth International Conference on Knowledge Representation and Reasoning (KR'06), pp. 210-220 (2006)

9. de Saint-Cyr, F.D., Lang, J.: Belief extrapolation (or how to reason about observations and unpredicted change). In: Proceedings of the Eighth Conference on Principles of Knowledge Representation and Reasoning (KR'02), pp. 497-508 (2002) 
10. Gärdenfors, P.: Knowledge in Flux. MIT Press (1988)

11. Gärdenfors, P., Makinson, D.: Revisions of knowledge systems using epistemic entrenchment. In: Proceedings of the Second Conference on Theoretical Aspects of Reasoning about Knowledge, pp. 83-95 (1988)

12. Grove, A.: Two modellings for theory change. J. Philos. Logic 17, 157-170 (1988)

13. Hansson, S.O. (ed.): Theoria. Special Issue on Non-Prioritized Belief Revision, vol. 63, nos. 1-2. Wiley (1997)

14. Herzig, A., Rifi, O.: Update operations: a review. In: Proceedings of the Thirteenth European Conference on Artificial Intelligence (ECAI'98), pp. 13-17 (1998)

15. Herzig, A., Rifi, O.: Propositional belief base update and minimal change. Artif. Intell. 115(1), 107-138 (1999)

16. Katsuno, H., Mendelzon, A.O.: Propositional knowledge base revision and minimal change. Artif. Intell. 52, 263-294 (1991)

17. Katsuno, H., Mendelzon, A.O.: On the difference between updating a knowledge base and revising it. In: Gärdenfors, P. (ed.) Belief Revision. Cambridge University Press (1992)

18. Konieczny, S.: Belief base merging as a game. J. Appl. Non-Class. Log. 14(3), 275-294 (2004)

19. Konieczny, S., Lang, J., Marquis, P.: DA² merging operators. Artif. Intell. 157(1-2), 49-79 (2004)

20. Konieczny, S., Pino Pérez, R.: Merging information under constraints: a logical framework. J. Log. Comput. 12(5), 773-808 (2002)

21. Konieczny, S., Pino Pérez, R.: Logic based merging. J. Philos. Logic 40(2), 239-270 (2011)

22. Konieczny, S., Pino Pérez, R.: Confluence operators. In: Proceedings of the Eleventh European Conference on Logics in Artificial Intelligence (JELIA'08), pp. 272-284 (2008)

23. Lang, J.: Belief update revisited. In: Proceedings of the International Joint Conference on Artificial Intelligence (IJCAI'07), pp. 2517-2522 (2007)

24. Lehmann, D., Magidor, M., Schlechta, K.: Distance semantics for belief revision. J. Symb. Log. 66(1), 295-317 (2001)

25. Liberatore, P., Schaerf, M.: Arbitration (or how to merge knowledge bases). IEEE Trans. Knowl. Data Eng. 10(1), 76-90 (1998)

26. Lin, J., Mendelzon, A.O.: Merging databases under constraints. Int. J. Cooperative Inf. Syst. 7(1), 55-76 (1998)

27. Meyer, T., Foo, N., Zhang, D., Kwok, R.: Logical foundations of negotiation: Outcome, concession and adaptation. In: Proceedings of the American National Conference on Artificial Intelligence (AAAI'04), pp. 293-298 (2004)

28. Meyer, T., Foo, N., Zhang, D., Kwok, R.: Logical foundations of negotiation: Strategies and preferences. In: Proceedings of the Ninth Conference on Principles of Knowledge Representation and Reasoning (KR'04), pp. 311-318 (2004)

29. Revesz, P.Z.: On the semantics of arbitration. Int. J. Algebra Comput. 7(2), 133-160 (1997)

30. Schlechta, K., Lehmann, D., Magidor, M.: Distance semantics for belief revision. In: Proceedings of: Theoretical Aspects of Rationality and Knowledge, Tark VI, pp. 137-145 (1996)

31. Tversky, A., Kahneman, D.: Extensional vs. intuitive reasoning: the conjunction fallacy in probability judgment. Psychol. Rev. 91(4), 293-315 (1983)

32. Zhang, D., Foo, N., Meyer, T., Kwok, R.: Negotiation as mutual belief revision. In: Proceedings of the American National Conference on Artificial Intelligence (AAAI'04), pp. 317-322 (2004) 